\title{
nsROC: An R package for Non-Standard ROC Curve Analysis
}

by Sonia Pérez-Fernández, Pablo Martínez-Camblor, Peter Filzmoser and Norberto Corral

\begin{abstract}
The receiver operating characteristic (ROC) curve is a graphical method which has become standard in the analysis of diagnostic markers, that is, in the study of the classification ability of a numerical variable. Most of the commercial statistical software provide routines for the standard ROC curve analysis. Of course, there are also many $\mathrm{R}$ packages dealing with the ROC estimation as well as other related problems. In this work we introduce the nsROC package which incorporates some new ROC curve procedures. Particularly: ROC curve comparison based on general distances among functions for both paired and unpaired designs; efficient confidence bands construction; a generalization of the curve considering different classification subsets than the one involved in the classical definition of the ROC curve; a procedure to deal with censored data in cumulative-dynamic ROC curve estimation for time-to-event outcomes; and a non-parametric ROC curve method for meta-analysis. This is the only $\mathrm{R}$ package which implements these particular procedures.
\end{abstract}

\section{Introduction}

Given a continuous variable, (bio)marker, we are frequently interested in performing a binary classification according to its value. This binary classification can be regarded as the presence or not of a certain characteristic of interest in the population (for instance, one disease). On the basis of data containing the real diagnosis, subjects are called positive when they have the characteristic and negative otherwise.

The receiver operating characteristic (ROC) curve assumes that higher values of the marker are associated with a higher probability of having the characteristic. Therefore, a subject whose marker value is below a fixed point (usually called threshold or cut-off point) is classified as negative (without the characteristic) while a subject with a marker value above the threshold is classified as positive (with the characteristic). Under this proviso, it displays the ability of the marker to correctly classify a positive subject as positive, or true-positive rate (TPR), versus the inability to correctly classify a negative subject as negative, or false-positive rate (FPR), for each cut-off point along all the possible values of the marker. That is, the sensitivity (TPR) versus the complementary of the specificity (FPR) for each possible threshold. In addition, the area under the ROC curve, AUC, is frequently used as an index of the global diagnostic capacity (Fluss et al., 2005). It ranges between $1 / 2$, when the marker does not contribute to a correct classification, and 1, if the marker may classify subjects properly. If $\mathrm{AUC}$ is less than $1 / 2$ it means that the direction of the classification should be the opposite (see comments about side of the ROC curve discussed below).

Mathematically, let $\chi$ and $\xi$ be two continuous random variables representing the marker values for negative and positive subjects, respectively. For a fixed value $t \in[0,1]$, the usual ROC curve (right-sided) can be defined as follows in terms of the distribution function of negative $\left(F_{\chi}\right)$ and positive $\left(F_{\xi}\right)$ group:

$$
\mathcal{R}(t)=1-F_{\mathcal{\zeta}}\left(F_{\chi}^{-1}(1-t)\right)=F_{1-F_{\chi}(\xi)}(t)
$$

leading the following area under the curve:

$$
\mathcal{A}=\int_{0}^{1} \mathcal{R}(t) d t=\mathcal{P}(\chi<\xi)
$$

Of course there exists a wide literature dealing with both theoretical and practical aspects of the ROC curve and other related problems. The interested reader can consult the monographs of Zhou et al. (2002) and Pepe (2003) for an extensive review of the topic. There are also a number of papers dealing with some problems related to ROC curve such as the usual ROC curve point estimation (see Gonçalvez et al. (2014) for a recent overview) from both parametric and non-parametric approaches, even considering Bayesian methods as an alternative to the maximum likelihood principle; or the curve interval estimation (confidence bands construction) also using both parametric (Demidenko, 2012) and non-parametric techniques (Jensen et al. (2000), Horváth et al. (2008) and Martínez-Camblor et al. (2018)).

Furthermore, the ROC curve procedure has been extended to other situations where the outcome is not binary. For instance, Mossman (1999) extends ROC concepts to diagnostic tests with trichotomous outcomes; whereas Heagerty and Zheng (2005) deal with time-dependent responses, whose most direct extension is by means of the cumulative/dynamic approach (Heagerty et al., 2000), but it 
involves a new problem: handling censored data. Additionally, Martínez-Camblor et al. (2017) proposed a ROC curve generalization for non-monotone relationships between the marker and the response, particularly convenient for situations in which both lower and higher marker values are associated with higher probabilities of having the studied characteristic. Some other scenarios where the information is not provided as standard may lead us to conduct a meta-analysis of ROC curves (see Martínez-Camblor (2017) and references therein) or fit a regression model for these curves (Cai (2004) and Rodríguez-Álvarez et al. (2011)).

On the other hand, the ROC curve comparison is one of the issues which has been more treated in literature. Usually ROC curves are compared from their respective AUCs, but in some situations these hypothesis tests are not the most appropriate (further discussed in the Comparison section). The similarity between two ROC curves have been traditionally discussed by Venkatraman and Begg (1996) for both paired and unpaired designs (Venkatraman, 2000). On the other hand, the comparison of the curves as functions is not different from the cumulative distribution function comparison problem, and this analogy was used by Martínez-Camblor et al. (2011), and subsequently extended to paired structures (Martínez-Camblor et al., 2013).

Some of the previous approaches have already been implemented in several software packages, including R packages such as pROC (Robin et al., 2018) and ROCR (Sing et al., 2015) which include different procedures to estimate the usual ROC curve (incorporating smoothing techniques), as well as confidence intervals computation for different parameters of the curve (sensitivity, specificity, AUC) and comparison of areas under two curves. There exist also more specific packages to deal with different particular topics and approaches of the ROC curve. For instance plotROC (Sachs, 2018) displays sophisticated plots of these curves; fbroc (Peter, 2016) focuses on a fast implementation of bootstrap techniques; OptimalCutpoints (Lopez-Raton and Rodriguez-Alvarez, 2014) includes several methods to select optimal cut-off points of the marker; timeROC (Blanche, 2015) and survivalROC (Heagerty and packaging by Paramita Saha-Chaudhuri, 2013) estimate time-dependent ROC curves and deal with some related analyses; and HSROC (Schiller and Dendukuri, 2015) implements a model for joint meta-analysis of sensitivity and specificity of the diagnostic test under evaluation.

ROC curves research is in fact a growing field in statistics. The aforementioned $\mathrm{R}$ packages are some of the most relevant ones in this topic but there are also more implementations covering certain algorithms. However, some non-standard ROC curve analyses exist which were not available to the scientific community in a practical software and this is the main reason why the new package presented in this paper has been created. The nsROC package (Pérez-Fernández, 2018) is a compilation of different analyses not computed to date which attempts to boost awareness of new techniques that have already been published but not implemented in a user-friendly software widely available. Furthermore, it incorporates several studies and techniques (from comparison of ROC curves to time-dependent estimation and meta-analysis), making it more manageable since all of them are included in the same package.

The rest of the paper is organized as follows: in the next two sections, Estimation and Comparison, some basic information about the statistical techniques included in the nsROC package, as well as some remarkable technical issues about its main functions, are provided. Particularly, the Estimation section incorporates several aforementioned situations: the ROC curve generalization for non-monotone relationships, confidence bands construction, censored data treatment for time-dependent outcomes, and meta-analysis involving ROC curves. In turn, the Comparison section includes three different methods of comparison (based on AUC, diagnostic capacity of the marker, or ROC curve definition in terms of CDF) to deal with both paired and unpaired data scenarios. Subsequently, in the Examples section, a complete analysis with different datasets is carried out to illustrate certain applications of the submitted package; and finally a Summary of the utility of the package is reported.

\section{Estimation}

\section{Non-standard ROC curve estimation}

As mentioned previously, an ROC curve is a graphical method which displays the sensitivity (Se) versus the complementary of the specificity (1-Sp) for all possible thresholds of the considered marker.

Although different parametric and semi-parametric estimators for the ROC curve have been studied, in our package the empirical estimator, based on replacing the involved unknown distribution functions with their respective empirical cumulative distribution functions, $\hat{F}$, has been considered. Hence, the implemented ROC curve estimator is

$$
\widehat{\mathcal{R}}(t)=\hat{F}_{1-\hat{F}_{\chi}(\xi)}(t) .
$$


This is the usual definition when higher values of the marker are considered to be associated with a higher probability of existence of the characteristic under study. It can be also called right-sided ROC curve.

However, sometimes it can be supposed the opposite, i.e. that higher values of the marker are associated with a lower probability of the existence of the characteristic. In this context, the definitions should be adapted and the resulting ROC curve (usually called left-sided curve) estimator is

$$
\widehat{\mathcal{R}}(t)=\hat{F}_{\hat{F}_{\chi}(\xi)}(t) .
$$

There exist several $\mathrm{R}$ packages also incorporating the non-parametric estimation, for instance the pROC package includes smoothed estimates. However, they suppose one of the assumptions aforementioned (right-sided or left-sided curve), considering a single threshold of the marker in order to classify, since the standard ROC curve definition is associated with this particular type of classification subsets.

Nevertheless, an extension of those classification subsets has been studied by Martínez-Camblor et al. (2017), dealing with situations in which not only higher or lower values of the marker are associated with a higher probability of existence of the studied characteristic, but both may be related. Under this assumption, not only one cut-off point is considered, but two $x_{l}$ and $x_{u}$ corresponding to the extremes of a marker interval are regarded, i.e those subjects with a marker value within the interval $\left(x_{l}, x_{u}\right)$ are classified as negative and those with a marker value below $x_{l}$ or greater that $x_{u}$ are supposed to be positive. In this context, the sensitivity and specificity definitions are the following ones:

$$
\begin{gathered}
S e\left(x_{l}, x_{u}\right)=P\left(\xi \leq x_{l} \cup \xi \geq x_{u}\right)=F_{\xi}\left(x_{l}\right)+1-F_{\xi}\left(x_{u}\right) \\
S p\left(x_{l}, x_{u}\right)=P\left(x_{l}<\chi<x_{u}\right)=F_{\chi}\left(x_{u}\right)-F_{\chi}\left(x_{l}\right) .
\end{gathered}
$$

At this juncture, it is important to note that there may be different couples $\left(x_{1}, x_{u}\right)$ reporting the same specificity but different sensitivity, so the generalized ROC curve is defined by the supreme of them:

$$
\mathcal{R}_{g}(t)=\sup _{\left(x_{l}, x_{u}\right) \in \mathcal{F}_{t}}\left\{F_{\mathcal{\xi}}\left(x_{l}\right)+1-F_{\mathcal{\xi}}\left(x_{u}\right)\right\}
$$

where $\left(x_{l}, x_{u}\right) \in \mathcal{F}_{t}$ iff $x_{l} \leq x_{u}$ and $S p\left(x_{l}, x_{u}\right) \geq 1-t$. It is clear that $\left(x_{l}, x_{u}\right) \in \mathcal{F}_{t}$ can also be written as $x_{l}=F_{\chi}^{-1}(\gamma t)$ and $x_{u}=F_{\chi}^{-1}(1-[1-\gamma] t)$ for some $\gamma \in[0,1]$, therefore

$$
\mathcal{R}_{g}(t)=\sup _{\gamma \in[0,1]}\left\{F_{\xi}\left(F_{\chi}^{-1}(\gamma t)\right)+1-F_{\xi}\left(F_{\chi}^{-1}(1-[1-\gamma] t)\right)\right\} .
$$

Using the aforementioned notation, the implemented general ROC curve estimator is

$$
\widehat{\mathcal{R}}_{g}(t)=\sup _{\gamma \in[0,1]}\left\{1-\hat{F}_{1-\hat{F}_{\chi}(\xi)}(1-\gamma t)+\hat{F}_{1-\hat{F}_{\chi}(\xi)}([1-\gamma] t)\right\} .
$$

Different parametric models have been considered in order to estimate the ROC curve. Among them, the binormal model is one of the most used, according to which the usual and general ROC curves, respectively, are the following:

$$
\begin{gathered}
\mathcal{R}(t)=\Phi\left(a+b \cdot \Phi^{-1}(t)\right) \\
\mathcal{R}_{g}(t)=\sup _{\gamma \in[0,1]}\left\{\Phi\left(a+b \cdot \Phi^{-1}([1-\gamma] \cdot t)\right)+1-\Phi\left(a+b \cdot \Phi^{-1}(1-\gamma \cdot t)\right)\right\}
\end{gathered}
$$

where $a=\left(\mu_{\xi}-\mu_{\chi}\right) / \sigma_{\xi}, b=\sigma_{\chi} / \sigma_{\xi}$ and $\Phi$ is the cumulative distribution function of a standard normal. Therefore, the parametric ROC curve estimation gets boiled down to estimate the parameters involved.

While the usual AUC has a direct probabilistic interpretation: "given two randomly and independently selected subjects, one negative and one positive, the AUC is the probability that the marker value in the positive subject is greater than in the negative subject", this reading is not directly related to the classification subsets involved in the definition of the usual ROC curve. However, it is possible to enunciate this relationship in terms of the diagnostic rule involved (citing Martínez-Camblor and Pardo-Fernández (2017)) and following the same idea the authors also proved the interpretation of the generalized AUC in terms of the probability of belonging to the corresponding classification subsets, under a condition about the continuity of $R_{g}(\cdot)$ and self-contained subsets as specificity increases.

In the nsROC package the point non-parametric ROC curve estimation can be computed by the gROC function. Some computational details must be mentioned: if Ni is NULL a fast algorithm is used to 
estimate the ROC curve for the considered sample; otherwise, if $\mathrm{Ni}$ is a number, thresholds considered are the marker values collected (adding $-\infty$ and $\infty$ ) and the specificities, $t$, used to estimate the ROC curve are those resulting from dividing the unit interval in Ni subintervals with the same length. This latter case is slower because the vector of $\gamma$-values taken into account in order to estimate the general ROC curve is the result of dividing the unit interval in subintervals with length 0.001 . The area under the curve is computed by the trapezoidal rule.

\begin{tabular}{|c|c|}
\hline \multicolumn{2}{|r|}{ Input parameters } \\
\hline$x$ & Vector of marker values. \\
\hline D & Vector of response values. Two levels; if more, the two first ones are used. \\
\hline side & $\begin{array}{l}\text { Type of ROC curve. One of "right" (right-sided), "left" (left-sided), "auto" } \\
\text { (right or left-sided is automatically chosen so that AUC will be greater than 0.5) or } \\
\text { "both" (general). Default: "right". }\end{array}$ \\
\hline $\mathrm{Ni}$ & Number of subintervals of the unit interval considered to compute the curve. De- \\
\hline & $\begin{array}{l}\text { fault: NULL (which will use the fast algorithm considering as many subintervals } \\
\text { as number of positive subjects). }\end{array}$ \\
\hline \multirow{3}{*}{$\begin{array}{l}\text { pval. auc } \\
\text { B }\end{array}$} & If TRUE, a permutation test to test $H_{1}: A U C \neq 0$ is performed. \\
\hline & Number of permutations used for testing. Default: 500. \\
\hline & Output parameters \\
\hline controls, cases & Marker values of negative and positive subjects, respectively. \\
\hline points. coordinates & Matrix whose second and third columns correspond to coordinates where the \\
\hline & $\begin{array}{l}\text { ROC curve has a step in case of right or left-sided ROC curves. In the first column } \\
\text { there are the marker thresholds considered reporting these coordinates. }\end{array}$ \\
\hline pairpoints.coordinates & Matrix whose third and fourth columns correspond to coordinates where the ROC \\
\hline & $\begin{array}{l}\text { curve has a step in case of general ROC curves. The first and second columns } \\
\text { are the marker thresholds considered, } x_{l} \text { and } x_{u} \text {, respectively, reporting these } \\
\text { coordinates. }\end{array}$ \\
\hline roc & Vector of values of the ROC curve for each $t$ considered. \\
\hline auc & Area under the curve estimate. \\
\hline \multirow[t]{2}{*}{ pval.auc, Paucs } & p-value and different permutation AUCs if the hypothesis test is performed. \\
\hline & Additional functions to be passed \\
\hline plot & Plot the ROC curve estimate. \\
\hline print & Print some relevant information. \\
\hline
\end{tabular}

Table 1: The most relevant input and output parameters of the gROC function.

The point estimation of the curve is essential, but it is also important to have an idea of how relevant the underlying sample is in this estimation, i.e. the interval estimation: how to build confidence bands of the ROC curve. This problem has been addressed from different points of view, most of them based on point-wise confidence intervals for sensitivity and/or specificity instead of focusing on the curve as a function.

There are some $\mathrm{R}$ packages providing some kind of confidence regions: fbroc includes a function which computes regions for the right-sided curve but no information about the method used to build them is provided; plotROC displays 'rectangular confidence regions for the ROC curve'; and pROC computes square pointwise confidence bands of the AUC, thresholds, specificity, sensitivity and/or coordinates of an ROC curve.

A review of the performance of these methods has already been carried out by Macskassy et al. (2005) who pointed out the difficulty of translating methods for building pointwise confidence intervals into methods to obtain confidence bands. However, when the focus is the whole ROC curve, one should construct confidence bands, and just considering the 'band' obtained joining the pointwise confidence intervals does not provide a real confidence band with the desired confidence level, because the probability that one point of the curve will be outside this 'band' is higher.

In this package three different techniques dealing with the ROC curve itself have been computed. Namely, one parametric assuming the binormal model (Demidenko (2012)) and two non-parametric have been included (Jensen et al. (2000) and Martínez-Camblor et al. (2018)):

- Demidenko (2012) adapted the Working-Hotelling type confidence bands used in linear regression and proposed a method called ellipse-envelope. It should be noted that the ROC curve estimated by this method is not empirical, but the binormal one.

- Jensen et al. (2000) approach is based on the asymptotic distribution of the ROC curve in terms of Brownian bridges, developing symmetrical non-parametric confidence bands for the curve, even on a particular region. The main drawback is the need to estimate density functions from smooth procedures involving a scale parameter (not chosen by the user) which can strongly 
affect the resulting ROC curve estimate. In terms of computational aspects it must be pointed out that the BBridge function in the sde (Iacus, 2016) package has been used to simulate the Brownian bridges involved. In addition, the extremes of the interval in $(0,1)$ in which the user wants to compute the regional confidence bands must be set. The bootstrap method has been applied and the confidence bands are truncated making the lower-band being inside the $(0,0.95)$ interval and the upper-band within $(0.05,1)$.

- Martínez-Camblor et al. (2018) method approximates the distribution of the following pivotal function by a smoothed bootstrap method:

$$
\sqrt{n} \cdot \sigma_{n}^{-1}(t) \cdot[\widehat{\mathcal{R}}(t)-\mathcal{R}(t)]
$$

where $n$ is the number of positive subjects and $\sigma_{n}(t)$ is the standard deviation estimate of $\sqrt{n}[\widehat{\mathcal{R}}(t)-\mathcal{R}(t)]$. Computational issues which should be taken into account are the following: confidence bands are truncated as in the previous method and the scale parameter, $s$, used to compute the smoothed kernel distribution functions (with bandwidth $h=s \cdot \hat{\sigma} \cdot \min \left\{n_{+}, n_{-}\right\}$) must be set by the user. Furthermore, there exists the option of selecting a parameter, $\alpha_{1}$, affecting the width between lower (and consequently upper) band and ROC curve point estimate. If $\alpha_{1}$ is not specified by the user, the one minimizing the theoretical area between the bands is automatically considered. It should be remarked that this is the only method designed to estimate ROC curve confidence bands for the general ROC curve.

\begin{tabular}{|c|c|}
\hline \multicolumn{2}{|r|}{ Input parameters } \\
\hline \multirow[t]{2}{*}{$\begin{array}{c}\text { conf.level } \\
\text { B } \\
\text { alpha1,s } \\
\text { a.J,b.J } \\
\text { plot.var }\end{array}$} & $\begin{array}{l}\text { Output of the gROC function. Ni is the number of subintervals used for estimation. } \\
\text { Method used. One of "PSN" (Martínez-Camblor et al., 2018), "JMS" (Jensen et al., 2000) on } \\
\text { "DEK" (Demidenko, 2012). } \\
\text { Confidence level considered. Default: } 0.95 \text {. } \\
\text { Number of bootstrap replicates. Default: } 500 \text {. } \\
\text { Parameters to pass to "PSN" method. Default: } s=1 \text {. } \\
\text { Extremes of interval to pass to "JMS" method. Default: a. J= } 1 / \mathrm{Ni}, \mathrm{b} . \mathrm{J}=1-1 / \mathrm{Ni} \text {. } \\
\text { If TRUE, variance estimate along } t \text { resulting from "PSN" or "JMS" method is displayed. }\end{array}$ \\
\hline & Output parameters \\
\hline \multirow[t]{2}{*}{$\begin{array}{l}\mathrm{L}, \mathrm{U} \\
\text { practical.area } \\
\text { alpha1, alpha2 }\end{array}$} & $\begin{array}{l}\text { Lower and upper bands respectively for each } t \in\{0,1 / \mathrm{Ni}, 2 / \mathrm{Ni}, \ldots, 1\} \text {. } \\
\text { Estimated area between lower and upper band. } \\
\alpha_{1} \text { and } \alpha_{2} \text { used in "PSN" method. }\end{array}$ \\
\hline & Additional functions to be passed \\
\hline $\begin{array}{l}\text { plot } \\
\text { print }\end{array}$ & $\begin{array}{l}\text { Plot the confidence bands of the ROC curve. } \\
\text { Print some relevant information. }\end{array}$ \\
\hline
\end{tabular}

Table 2: The most relevant input and output parameters of the Rocbands function.

\section{Time-dependent ROC curve}

Sometimes the response variable is not binary but time-dependent. In this case the resulting curve is called time-dependent ROC curve. Although there exist different approaches of this kind of curves depending on the association between the referred time-dependent outcome and the binary classification (for instance, Heagerty and Zheng (2005) considered the incident sensitivity defined as $S e^{I}(x)=P(X>x \mid T=t)$ to build the incident/dynamic ROC curve), the most direct one is the cumulative/dynamic approach, which classifies as positive a subject in which the event happens before a fixed point of time $t$ and negative otherwise. In other words, the cumulative sensitivity and the dynamic specificity are defined as follows: $S e^{C}(x)=P(X>x \mid T \leq t)$ and $S p^{D}(x)=P(X \leq x \mid T>t)$.

However, the time-dependent problem involves a new issue to be addressed: how to deal with subjects censored before $t$. There are some $\mathrm{R}$ packages which incorporate time-dependent ROC curve estimation procedures in the presence of censored data. Some good examples are timeROC, which also performs some estimations about different concepts related to time-dependent ROC curve and compare time-dependent AUCs (see Blanche et al. (2013) for a complete overview of the implemented methods); survivalROC which computes time-dependent ROC curves from censored survival data using the Kaplan-Meier (KM) or Nearest Neighbor Estimation (NNE) method by Heagerty et al. (2000); and tdROC (Li et al., 2016), based on the Li et al. (2018) method mentioned below.

In order to deal with time-dependent outcomes, the nsROC package has used the cumulative/dynamic approach. A different solution for the censoring problem has been proposed by MartínezCamblor et al. (2016), considering a time-dependent ROC curve estimator based on assigning a 
probability to be negative (consequently positive) to those censored subjects. Particularly, two different statistics have been suggested in order to estimate the probability of surviving beyond $t$ : a semiparametric one, using a proportional hazard Cox regression model considering the marker as the covariate; and a non-parametric one, using directly the Kaplan-Meier estimator. There exists a subsequent paper based on the same idea (Li et al., 2018) but using the kernel-weighted Kaplan-Meier estimator instead of the naive one. This last method is also included in nsROC package, allowing the user to choose the kernel and bandwidth to be considered in the kernel-weighted statistic.

In terms of computational aspects it should be noted that the survival (Therneau, 2018) package has been used. In particular, the survfit and Surv functions are required to estimate survival functions, and the coxph function is used to fit the Cox proportional hazard regression model involved in the semiparametric approach aforementioned.

\begin{tabular}{|c|c|}
\hline \multicolumn{2}{|r|}{ Input parameters } \\
\hline $\begin{array}{l}\text { stime } \\
\text { status } \\
\text { marker } \\
\text { predict.time } \\
\text { method } \\
\text { kernel } \\
\text { h, kernel.fun } \\
\text { boot.n }\end{array}$ & $\begin{array}{l}\text { Vector of observed times. } \\
\text { Vector of status ( } 0 \text { if the subject is censored and } 1 \text { otherwise). } \\
\text { Vector of marker values. } \\
\text { Time point } t \text { considered. } \\
\text { Method to estimate the probability aforementioned. One of "Cox", "KM" or "wKM". } \\
\text { Procedure used to calculate kernel function if method is "wKM". One of "normal", } \\
\text { "Epanechnikov" or "other" (if the user defines a different one). } \\
\text { Bandwidth and kernel function used if method is "wKM" and kernel is "other". } \\
\text { Number of bootstrap samples considered. Default: } 100 \text {. }\end{array}$ \\
\hline & Output parameters \\
\hline $\begin{array}{l}\text { TPR, TNR } \\
\text { cutPoints } \\
\text { auc }\end{array}$ & $\begin{array}{l}\text { Vector of sensitivities and specificities estimates, respectively. } \\
\text { Vector of marker thresholds considered. } \\
\text { Area under the time-dependent ROC curve estimate. }\end{array}$ \\
\hline & Additional functions to be passed \\
\hline $\begin{array}{l}\text { plot } \\
\text { print }\end{array}$ & $\begin{array}{l}\text { Plot the time-dependent ROC curve estimate. } \\
\text { Print some relevant information. }\end{array}$ \\
\hline
\end{tabular}

Table 3: The most relevant input and output parameters of the cdROC function.

\section{Meta-analysis}

Meta-analysis is a popular statistical methodology for combining the results from multiple independent studies about the same topic. It allows us to know the state of the art, strengths and weaknesses of one considered topic, combining estimation effects from different independent comparable studies (Riley et al., 2010). However, the main particularity of meta-analysis is that only limited information is available from each study considered. There exist two different meta-analysis models depending on the consideration (or not) of the variability between studies: the fixed-effects model just considers the within-study variability whereas the random-effects model also takes into account the variability between studies (DerSimonian and Laird, 1986).

In the case that the target is the ROC curve, the goal of meta-analysis is combining the results from several independent studies performed by the same marker and characteristic of interest in a single outcome. Different methods to compute summary ROC curves have been introduced in order to determine the global diagnostic accuracy for both fixed-effects (Moses et al. (1993)) and random-effects model (Hamza et al. (2008), among others). Besides, the HSROC package implements the procedure of Rutter and Gatsonis (2001). However, most of those approaches are parametric and consider that only one estimated pair of sensitivity and specificity from each paper exist and they are supposed to be independently selected in each study, but often the reported points are the best ones in the Youden index sense. Nevertheless, some new techniques have been developed taking into account all the pairs of points reported; Hoyer and Kuss (2018) and Steinhauser et al. (2016) are good examples. Martínez-Camblor (2017) includes a different view focusing on the direct ROC curve estimation from a non-parametric approach, using weighted means of each individual ROC curve, taking all pairs of points $(S e, S p)$ reported in each study, and performing a simple linear interpolation between them. Moreover, both the fixed and random-effects model are covered.

The metaROC function in the nsROC package implements this last approach reporting a fully nonparametric ROC curve estimate from a data frame including the number of true positive and negative (TP and TN) subjects, false positive and negative (FP and FN) subjects and a identifier of the study they come from. It displays in a plot the non-parametric summary ROC (nPSROC) curve estimate, and the user has the possibility of including all ROC curve interpolations in the same graphic, as well 
as a confidence band estimate. In the random-effects model there is also the option of plotting the inter-study variability estimate along the different specificities on the unit interval.

\begin{tabular}{|c|c|}
\hline \multicolumn{2}{|r|}{ Input parameters } \\
\hline data & A data frame containing the variables: "Author", "TP", "TN", "FP" and "FN". \\
\hline model & Meta-analysis model considered. One of "fixed-effects" or "random-effects". \\
\hline $\mathrm{Ni}$ & Number of subintervals of the unit interval considered to compute the curve. Default: 1000 . \\
\hline plot. Author & $\begin{array}{l}\text { If TRUE, a plot including ROC curve estimates (by linear interpolation) for each study under } \\
\text { consideration is displayed. }\end{array}$ \\
\hline plot.bands & If TRUE, confidence interval estimate for the ROC curve is added. \\
\hline plot.inter.var & If TRUE, a plot reflecting inter-study variability estimate is displayed on an additional window. \\
\hline \multicolumn{2}{|r|}{ Output parameters } \\
\hline sRA & $\begin{array}{l}\text { nPSROC curve estimate resulting from the model considered with a slight modification to } \\
\text { ensure the monotonicity along the points on the unit interval considered. }\end{array}$ \\
\hline se.RA & Standard-error of nPSROC curve estimate. \\
\hline area & Area under the curve estimate. \\
\hline youden. index & Optimal specificity and sensitivity in the Youden index sense for nPSROC curve. \\
\hline roc.j $\mathrm{j}$ & $\begin{array}{l}\text { A matrix whose columns contain the ROC curve estimate (by linear interpolation) of each } \\
\text { study. }\end{array}$ \\
\hline w.j,w.j.rem & $\begin{array}{l}\text { A matrix whose columns contain the weights in fixed or random-effects model, respectively, } \\
\text { of each study. }\end{array}$ \\
\hline
\end{tabular}

Table 4: The most relevant input and output parameters of the metaROC function.

\section{Comparison}

An important role of diagnostic medicine research is the comparison of the accuracy of diagnostic tests. With the goal of comparing their global accuracy, the comparison of AUCs is the most usual method (DeLong et al., 1988). However, when there is no uniform dominance between the involved curves (i.e. the sensitivities associated with each specificity along the unit interval are not always higher in one curve than in the other), they can differ having the same AUC. In these situations, these tests are not valid to compare the equality among the ROC curves, and some other approaches could be considered to compare the equality of all the curves, such as Martínez-Camblor et al. (2013) and Martínez-Camblor et al. (2011) mentioned below, which deal with the ROC curve by its definition as a cumulative distribution function. On the other hand, Venkatraman and Begg (1996) and Venkatraman (2000) propose the use of a non-parametric permutation test to compare the equality of two diagnostic criteria. Both paired and unpaired designs have been treated, i.e. when different markers for detecting the existence of one characteristic are compared in the same sample (just one positive-negative sample) or when the same marker is compared along different and independent samples (as many positivenegative samples as groups to compare), respectively.

In the first case (paired design), different non-parametric tests have been implemented to perform the comparison:

- The procedure of Martínez-Camblor et al. (2013) takes into account the expression of the ROC curve in terms of the distribution function shown in the Estimation section and extends classical tests for comparing the cumulative distribution functions to this context. Four of these tests have been included in the compareROCdep function but any other can be defined by the FUN. dist input parameter. Those included are the following: Kolmogorov-Smirnov, the two ones based on the $L_{1}$ or $L_{2}$ measure and Cramér von-Mises. It is important to highlight that the user can set any other criteria to perform the test.

Two different methods could also be considered in order to approximate the distribution function of the selected statistic under the null hypothesis: the procedure of Venkatraman and Begg (1996) or the one of Martínez-Camblor and Corral (2012) based on permutated and bootstrap samples, respectively. This last one (gBA) is a novel bootstrap procedure which allows us to deal with complex structures.

- Venkatraman and Begg (1996) method tests the hypothesis that two curves are identical for all cut-off points. It should be noted that the permutation procedure covered in this paper requires the exchangeability assumption.

Some technical issues should be also indicated: if the comparison involves more than two ROC curves, the value of the statistic is the sum of the corresponding values of each pair without repetition. In addition, the Venkatraman estimator has been developed just for comparing right-sided ROC curves. 
- One test based on the comparison of the areas under the curve has also been included; in particular, the one proposed by DeLong et al. (1988). It should be noted that two different ROC curves can have the same AUC as it has been mentioned above. In computational terms this procedure takes longer because the statistic involved requires positive sample size $\times$ negative sample size comparisons.

\begin{tabular}{|c|c|}
\hline \multicolumn{2}{|r|}{ Input parameters } \\
\hline $\mathrm{x}$ & A matrix whose columns are the vectors of each marker-values sample. \\
\hline D & Vector of response values. \\
\hline side & Type of ROC curve. One of "right" or "left". \\
\hline statistic & $\begin{array}{l}\text { Statistic used to compare the curves. One of "KS", "L1", "L2", "CR", "other" (if the user defines } \\
\text { a different one using other input parameters) or "VK". }\end{array}$ \\
\hline FUN.dist & $\begin{array}{l}\text { The distance considered as a function of one variable. Example: FUN.dist }= \\
\text { function }(\mathrm{g})\{\max (\operatorname{abs}(\mathrm{g}))\} \text { defines the Kolmogorov-Smirnov statistic. }\end{array}$ \\
\hline method & $\begin{array}{l}\text { Method used to approximate the statistic distribution under the null. One of } \\
\text { "general.bootstrap", "permutation" or "auc". }\end{array}$ \\
\hline B, perm & Number of bootstrap or permutation samples considered, respectively. Default: 500 . \\
\hline plot.roc & If TRUE, a plot including the ROC curve estimates for each sample and their mean is displayed. \\
\hline \multicolumn{2}{|r|}{ Output parameters } \\
\hline statistic & The value of the test statistic. \\
\hline p.value & lue for the test. \\
\hline
\end{tabular}

Table 5: The most relevant input and output parameters of the compareRocdep function.

In the second case (unpaired design), different non-parametric tests have also been implemented to perform the comparison. They are similar to the previous ones:

- The comparisons of Martínez-Camblor et al. (2011) are inspired by the usual distances between cumulative distribution functions. Three of those distances have been included in the compareROCindep function (particularly, the two ones based on $L_{1}$ and $L_{2}$ measures and the Cramér von-Mises criterion), but it should be highlighted that the user has the possibility to define any other distance by the FUN. stat. dist and FUN. stat.cons input parameters, described in more detail below.

Furthermore, the permutation method proposed by Venkatraman (2000) is used to approximate the distribution function of the selected statistic under the null. Related to this method, both raw or ranked data (including a method for breaking ties) could be considered.

- The procedure of Venkatraman (2000) is based on the idea that two ROC curves are identical if and only if for every cut-off point from one marker there is an equivalent one from the other with the same probabilities of failure, i.e the same sensitivity and specificity. Technical issues which are worth noting are the same as those aforementioned in the Venkatraman method for paired samples.

A straightforward k-sample non-parametric test for the AUC statistic computing the differences with respect to the mean can also be considered. It should be remembered the consideration mentioned above about comparing areas under the curve.

\section{Examples}

Some examples analysing real data-sets are shown in this section in order to illustrate the application of the different functions included in the nsROC package. Namely, the Breast Cancer dataset is used to show the different estimation of the ROC curve considering the usual definition versus the generalization (gROC function) as well as confidence bands estimation reported by different procedures, particularly PSN, JMS and DEK (ROCbands function). Furthermore, a comparison of the ROC curve reported by two different markers is performed by the compareROCdep function and also the diagnostic capacity of one marker in three different groups is studied by the compareROCindep function. The intended goal of the Primary Biliary Cirrhosis dataset example is considered to show time-dependent ROC curve estimation in the presence of censored data at a specific time using different procedures implemented in cdROC function: Cox, KM and wKM with different kernels. Finally, the Interleukin 6 dataset includes the results regarding diagnostic ability of a marker over the same characteristic reported by different research papers and the goal is to perform a meta-analysis over them in order to unify the studies in one unique response (metaROC function). 


\begin{tabular}{|c|c|}
\hline \multicolumn{2}{|r|}{ Input parameters } \\
\hline $\mathrm{x}$ & Vector of marker values. \\
\hline G & Vector of group identifier values (with as many levels as independent samples to compare). \\
\hline D & Vector of response values. \\
\hline side & Type of ROC curve. One of "right" or "left". \\
\hline statistic & $\begin{array}{l}\text { Statistic used to compare the curves. One of "L1", "L2", "CR", "other" (if the user defines a } \\
\text { different one using other input parameters), "VK" or "AUC". }\end{array}$ \\
\hline FUN. stat.int & $\begin{array}{l}\text { A function of two variables, roc. } i \text { and roc standing for ROC curve estimate for } i \text {-th sample } \\
\text { and mean ROC curve estimate along k samples, respectively. Example: FUN. stat. int }= \\
\text { function }(r o c . i, r o c)\{\text { mean (abs (roc. } i-r o c) \text { ) }\} \text { defines the } L_{1} \text {-measure statistic. }\end{array}$ \\
\hline raw & $\begin{array}{l}\text { If TRUE, raw data is considered; if FALSE (default) data is ranked and a method to break ties } \\
\text { in permutations is performed. }\end{array}$ \\
\hline \multirow{3}{*}{$\begin{array}{l}\text { perm } \\
\text { plot.roc }\end{array}$} & Number of permutation samples considered. Default: 500 . \\
\hline & If TRUE, a plot of ROC curve estimates for each sample and their mean is displayed. \\
\hline & Output parameters \\
\hline statistic & The value of the test statistic. \\
\hline p.value & The p-value for the test. \\
\hline
\end{tabular}

Table 6: The most relevant input and output parameters of the compareROCindep function.

\section{Breast cancer dataset}

The Breast Cancer dataset consists of several features computed from a digitized image of a fine needle aspirate (FNA) of a breast mass describing the characteristics of the cell nuclei present in a 3D image. This dataset, freely available at https://archive.ics.uci.edu/ml/machine-learningdatabases/breast-cancer-wisconsin/wdbc.data, includes a diagnosis variable ("malignant" vs "benign") and ten real-valued features about each cell nucleus (radius, texture, perimeter, area, smoothness, compactness, concavity, concave points, symmetry and fractal dimension) collected from 569 patients in Wisconsin. The mean, standard error and worst (defined as the mean of the three largest values) of these features were computed for each image, resulting in 30 variables. The reader is referred to Bennett and Mangasarian (1992) for a complete information about the dataset.

There exists a variable, the fractal dimension (mean), which does not seem to correctly distinguish between "malignant" and "benign" cases, reporting an usual ROC curve (left-sided) crossing the diagonal with an AUC of 0.513. Looking at the density function estimates displayed in Figure 1 (top), it can be seen that although the vast majority of the fractal dimension values are in the interval $(0.055,0.075)$ in both groups (so this marker is not a good one to perform the classification), lower and higher values are likely to be "malignant" cases (positive subjects). Thus, it makes sense to compute the general ROC curve estimate proposed in this package, which reports an AUC of 0.633 , higher than the usual one, and of course the curve is above the diagonal by definition (see graph bottom-right in Figure 1).

Figure 1 and information about ROC curve estimates have been reported using the gROC function:

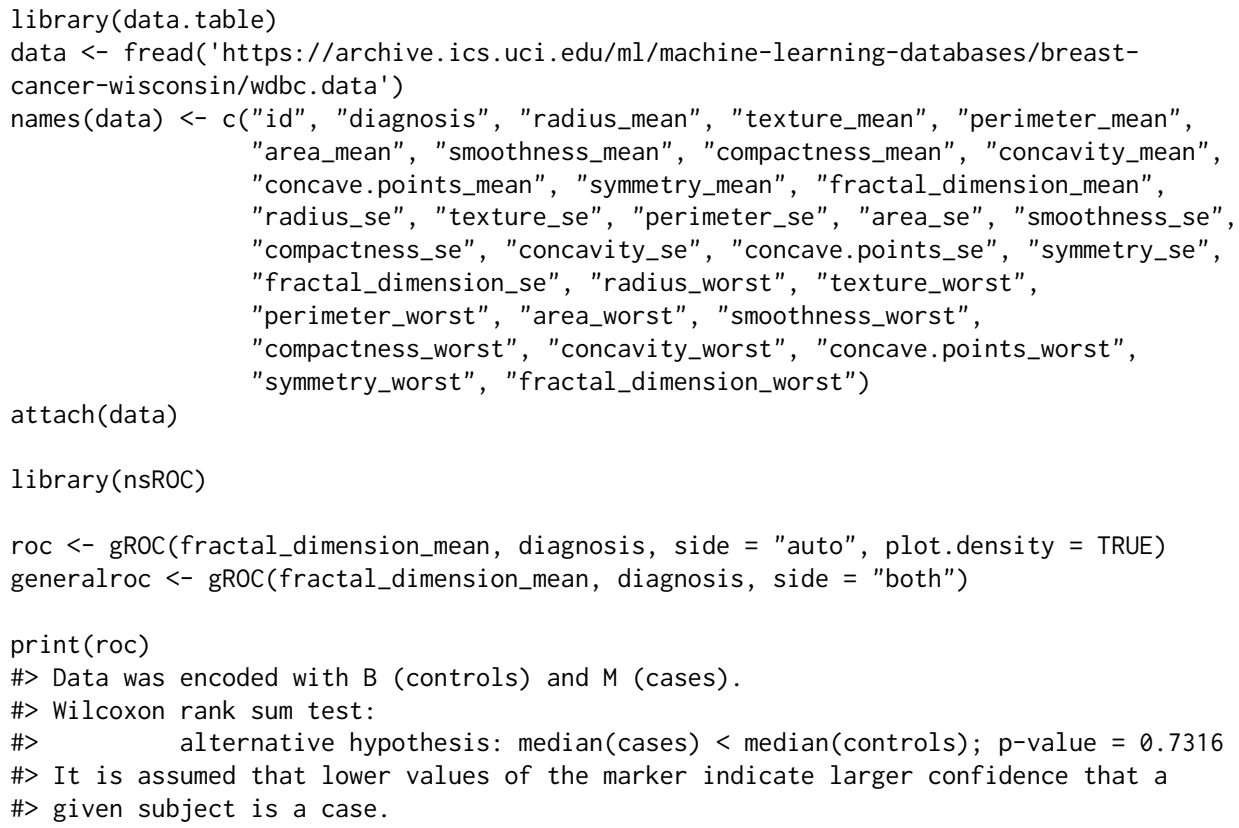




\section{Density estimation}

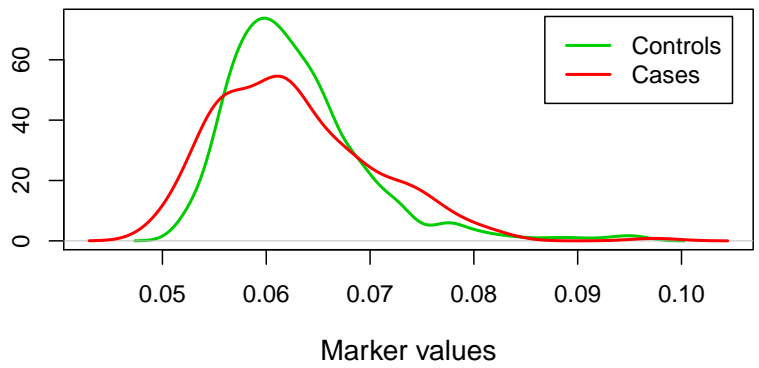

ROC curve (left-sided)

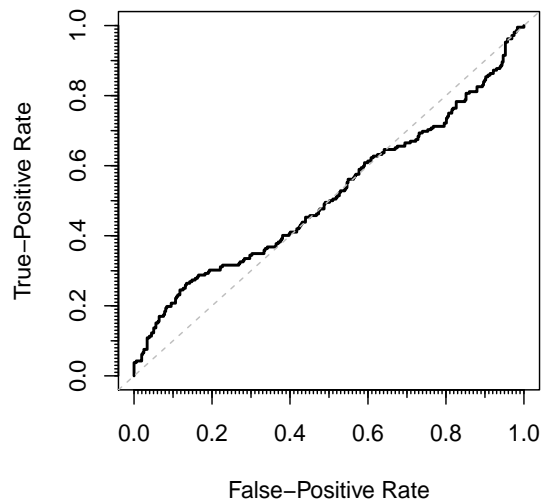

General ROC curve

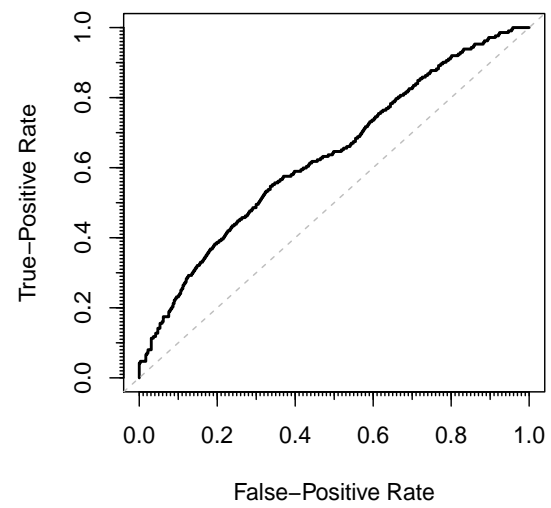

Figure 1: Top, density function estimates of fractal dimension mean variable for both "malignant" and "benign" subjects. Bottom, left-sided and generalized ROC curve estimates, respectively.

\#> There are 357 controls and 212 cases.

\#> The area under the ROC curve (AUC) is 0.513 .

print (generalroc)

\#> Data was encoded with B (controls) and M (cases).

\#> It is assumed that both lower and larges values of the marker indicate larger

\#> confidence that a given subject is a case.

\#> There are 357 controls and 212 cases.

\#> The area under the ROC curve (AUC) is 0.633 .

$\operatorname{plot}($ roc, main $=$ "ROC curve (left-sided)")

plot (generalroc, main = "General ROC curve")

In order to illustrate the confidence bands construction reported by each method implemented ("PSN" (Martínez-Camblor et al., 2018), "JMS" (Jensen et al., 2000) and "DEK" (Demidenko, 2012)), a marker with a better global diagnostic accuracy (in terms of AUC) than the previous one has been considered: the texture (mean).

In Figure 2 it can be seen that not only the bands are different but also the ROC curve point estimates. This is because each method uses a different way to compute it: "PSN" considers the same as the one computed in the gROC function, "JMS" performs a similar one with smoothed estimators and "DEK" computes a parametric estimate based on the assumption of the binormal model. This last one displays the narrowest confidence bands as it was expected (with an area between the CI bands equals to 0.069 ).

The ROCbands function has been used for this purpose:

roc $<-$ gROC(texture_mean, diagnosis)

\# right-sided in this case

rocbands_psn <- ROCbands (roc, method = "PSN")

rocbands_psn_mod <- ROCbands(roc, method = "PSN", alpha1 = 0.025)

rocbands_jms <- ROCbands (roc, method = "JMS")

rocbands_dek <- ROCbands (roc, method = "DEK") 

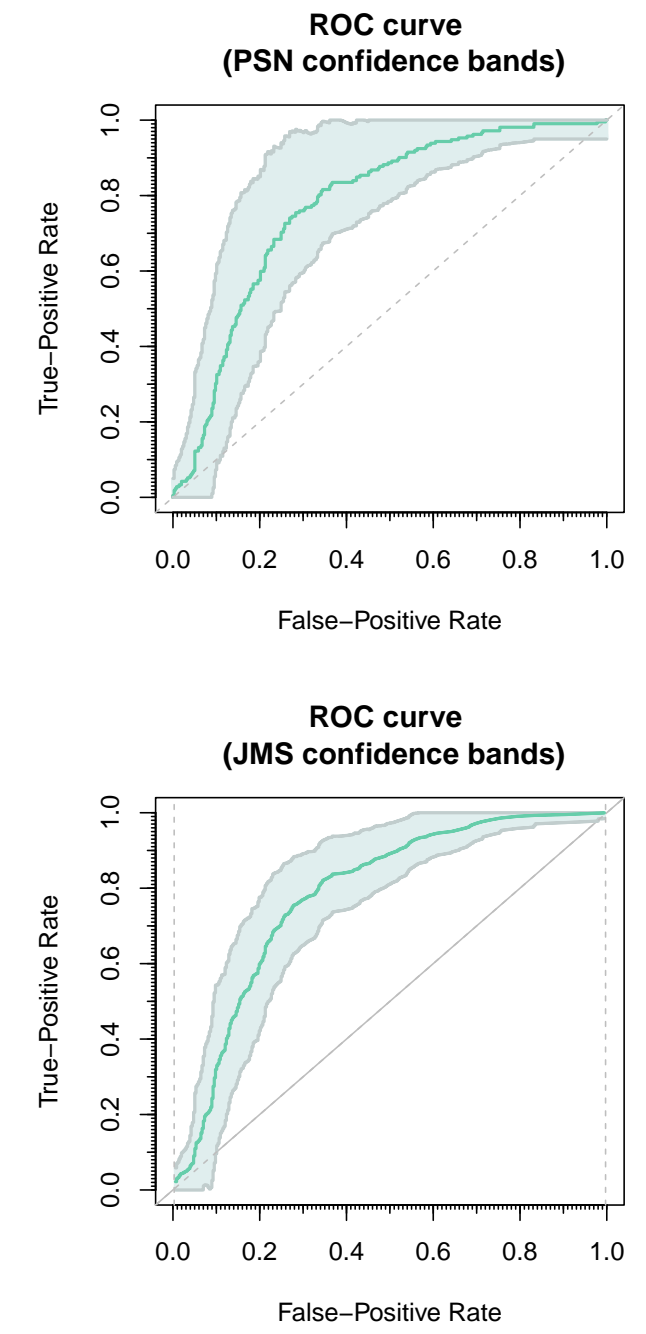
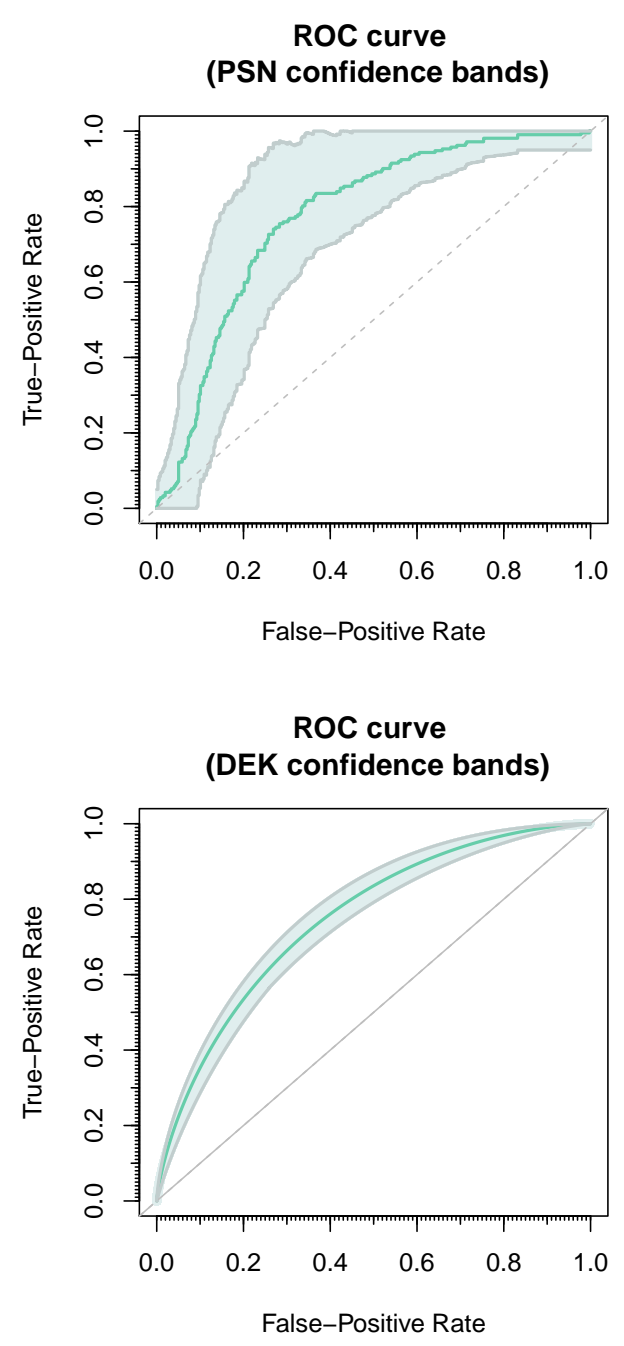

Figure 2: Top, confidence bands for the ROC curve using the "PSN" procedure for optimal $\alpha_{1}$ and fixed $\alpha_{1}=\alpha / 2$, respectively. Bottom, confidence bands for the ROC curve constructed by the "JMS" and "DEK" method, respectively. Confidence level: $1-\alpha=0.95$.

The computations performed to get the graphics and some useful information about the confidence bands construction are detailed below:

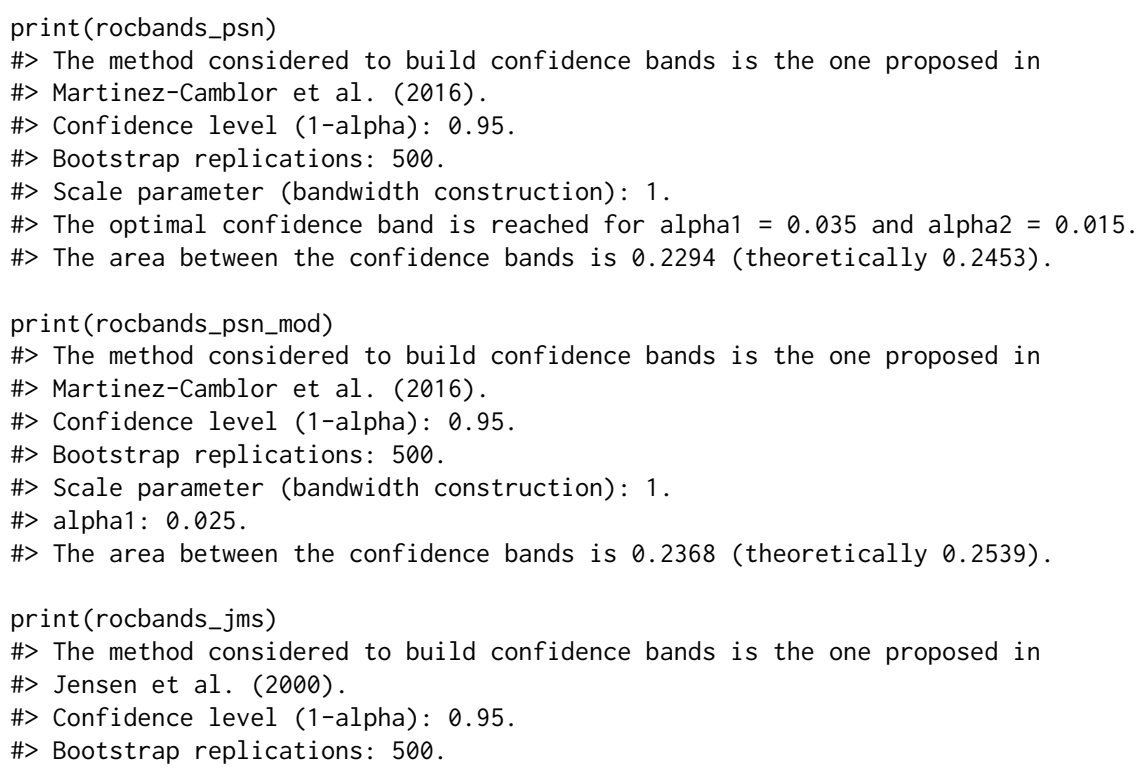


\#> Interval in which compute the regional confidence bands: $(0.00280112,0.9971989)$.

\#> K.alpha: 3.163202.

\#> The area between the confidence bands is 0.1668 .

print (rocbands_dek)

\#> The method considered to build confidence bands is the one proposed in

\#> Demidenko (2012).

\#> Confidence level (1-alpha): 0.95 .

\#> The area between the confidence bands is 0.0694 .

plot (rocbands_psn)

plot (rocbands_psn_mod)

plot (rocbands_jms)

plot (rocbands_dek)

Figure 3 presents the comparison of two dependent ROC curves describing the ability of the markers mean and the worst smoothness to make an accurate diagnosis. Different procedures dealing with different estimators and ways to approximate their distribution under the null hypothesis $\left(H_{0}: R_{1}(t)=R_{2}(t) \forall t \in(0,1)\right)$ have been considered.
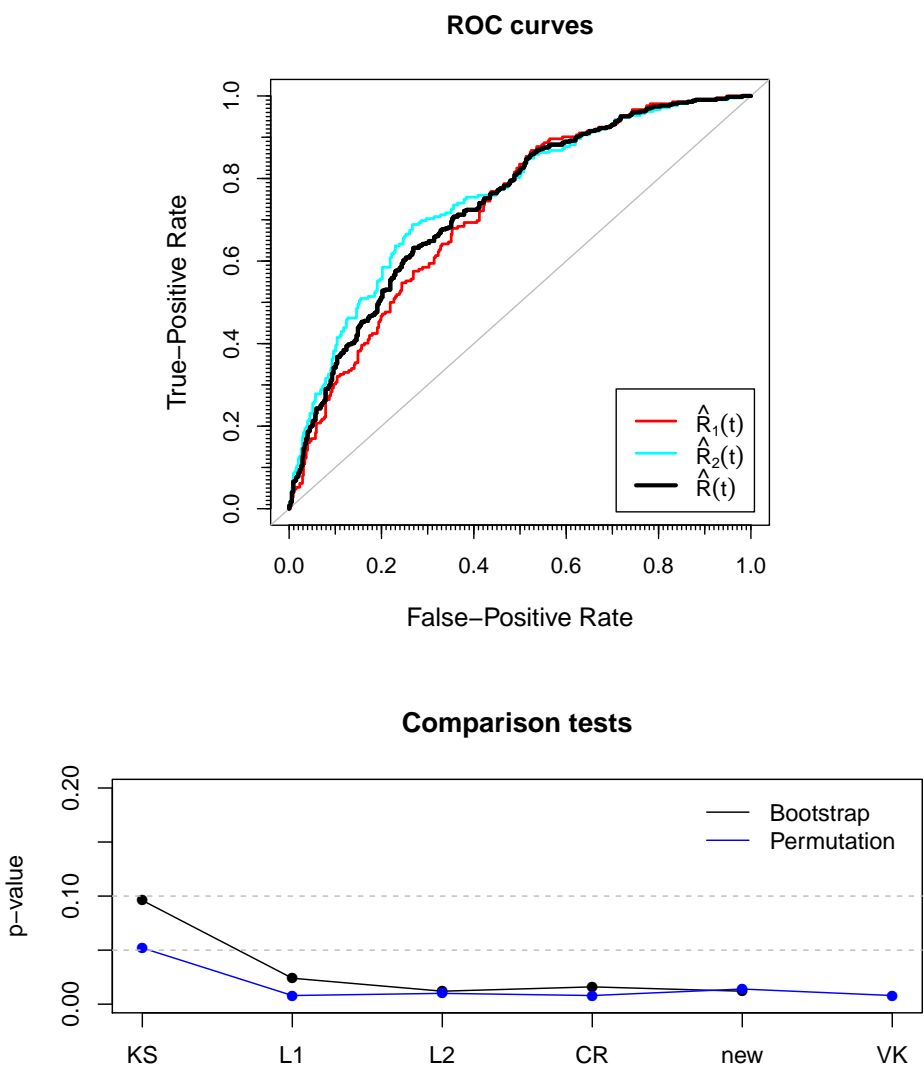

Figure 3: Top, ROC curve estimates for mean $\left(\widehat{R}_{1}(t)\right)$ and worst $\left(\widehat{R}_{2}(t)\right)$ smoothness, in black the mean ROC curve estimate $(\widehat{R}(t))$. Bottom, p-values of previous tests by bootstrap (black line) and permutated (blue line) iterations based on the Kolmogorov-Smirnov test, $L_{1}$ and $L_{2}$ measures, Cramér von-Mises criterion, a new one whose statistic value is defined as $\frac{1}{2} \sum_{i=1}^{2} \int_{0}^{1} n^{2}\left(\widehat{R}_{i}(t)-\widehat{R}(t)\right)^{4} d t$, and the Venkatraman approach.

The p-values reported by every method are below 0.05 except for Kolmogorov-Smirnov. It should be noted that the $\mathrm{p}$-values returned by the Venkatraman permutation method are slightly lower than those ones obtained by the general bootstrap technique.

The compareROCdep function has been used with this objective:

depmarker <- cbind(smoothness_mean, smoothness_worst) 
out.KS <- compareROCdep(depmarker, diagnosis)

out.L1 <- compareROCdep(depmarker, diagnosis, statistic = "L1")

out.L2 <- compareROCdep (depmarker, diagnosis, statistic = "L2")

out.CR <- compareROCdep (depmarker, diagnosis, statistic = "CR")

out.new <- compareROCdep (depmarker, diagnosis, statistic = "other", FUN.dist $=$ function $\left.(g)\left\{\operatorname{mean}\left(g^{\wedge} 4\right)\right\}\right)$

out.perm.KS <- compareROCdep(depmarker, diagnosis, method = "perm")

out. perm.L1 <- compareROCdep(depmarker, diagnosis, statistic = "L1", method = "perm")

out.perm.L2 <- compareROCdep(depmarker, diagnosis, statistic = "L2", method = "perm")

out. perm.CR <- compareROCdep(depmarker, diagnosis, statistic = "CR", method = "perm")

out.VK <- compareROCdep(depmarker, diagnosis, statistic="VK")

out. perm.new <- compareROCdep(depmarker, diagnosis, statistic = "other", method = "perm",

FUN.dist $=$ function $\left.(g)\left\{\operatorname{mean}\left(g^{\wedge} 4\right)\right\}\right)$

On the other hand, Figure 4 reflects the comparison of three independent ROC curves performed to analyze the diagnostic accuracy of the mean radius variable in each group defined by symmetry values: group 1 if symmetry_mean $<0.18$ and symmetry_worst $<0.29$, group 3 if symmetry_mean $>0.18$ and symmetry_worst $>0.29$, and group 2 otherwise. The five estimators computed in the compareROCindep function have been used.
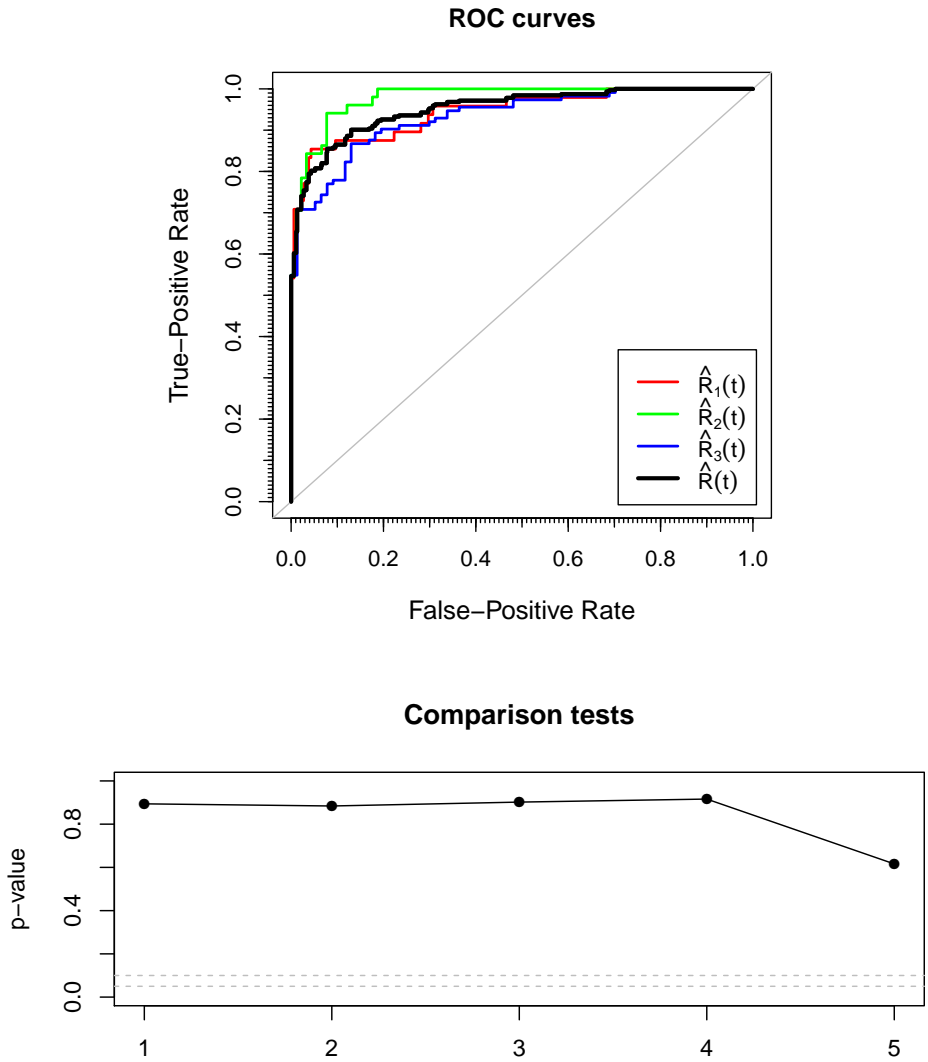

Figure 4: Top, ROC curve estimates for radius mean variable in each group $\left(\widehat{R}_{i}(t)\right)$ and their mean ROC curve estimate $(\widehat{R}(t))$. Bottom, p-values of previous tests based on $L_{1}$ and $L_{2}$ measures, Cramér von-Mises criterion, Venkatraman approach and AUC comparison test.

The p-value is greater than 0.1 for all tests considered, being the one reported by the AUC approach the lowest one. Therefore it might be concluded that there is no statistically significant evidence to state that these three ROC curves differ.

The commands used to build Figure 4 are the following, using the compareROCindep function:

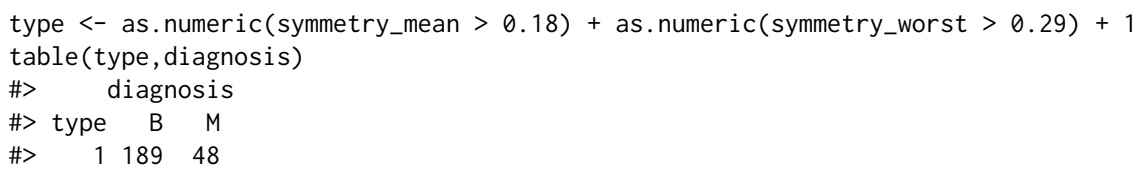


\#> $\quad 2 \quad 91 \quad 51$

\#> $\quad 3 \quad 77 \quad 113$

output.L1 <- compareROCindep(radius_mean, type, diagnosis, statistic = "L1") output.L2 <- compareROCindep(radius_mean, type, diagnosis, statistic = "L2") output.CR <- compareROCindep(radius_mean, type, diagnosis, statistic = "CR") output.VK <- compareROCindep(radius_mean, type, diagnosis, statistic = "VK") output.AUC <- compareROCindep(radius_mean, type, diagnosis, statistic = "AUC")

\section{Primary Biliary Cirrhosis (PBC) Data}

The Primary Biliary Cirrhosis (PBC) dataset contains the results of a trial in PBC of the liver conducted between 1974 and 1984 referred to Mayo Clinic. A total of 424 PBC patients met eligibility criteria for the randomized placebo controlled trial of the drug D-penicillamine; among them, the 393 nontransplanted ones have been considered for this analysis. This dataset is freely available within the $\mathrm{R}$ package survival by the name pbc. The reader is referred to Therneau and Grambsch (2000) for a complete information about the study.

In order to analyze how good the marker serum bilirubin $(\mathrm{mg} / \mathrm{dl})$ is to detect those patients who died or survived by 4000 days from their registration in the study, the ROC curve has been estimated. However, there are some patients censored before the regarded time, and two different approaches have been considered in order to estimate the survival probability of those patients censored before the time considered: Figure 5 at top-left, a semi-parametric one based on Cox regression model; at top-right and bottom, a non-parametric one based on naive and smoothed Kaplan-Meier estimators, respectively.

As shown in Figure 5, the different approaches considered report similar ROC curves but it should be noted that the area under the curve reported by the weighted Kaplan-Meier method with normal kernel is slightly higher $(\mathrm{AUC}=0.809)$ because the sensitivities related to specificity values close to one are the highest.

The cdROC function has been used for this purpose:

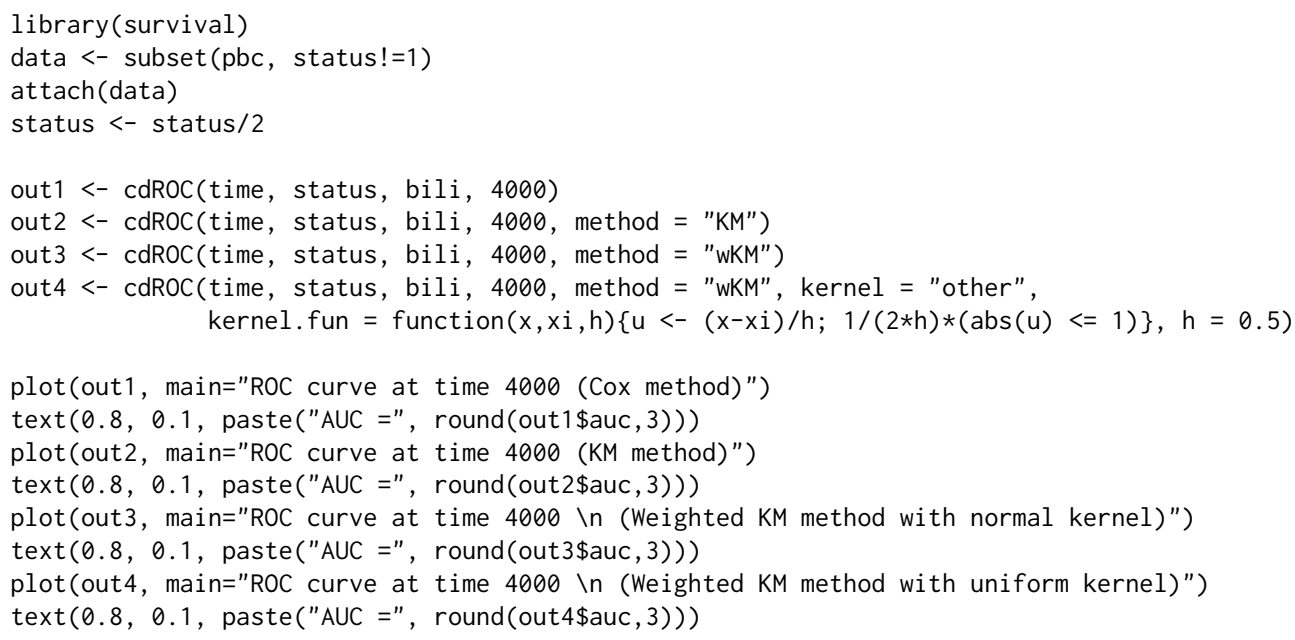

\section{Interleukin 6 (IL6) Data}

The Interleukin 6 (IL6) dataset includes the results of 9 papers which study the use of the IL6 as a marker for the early detection of neonatal sepsis. An analysis of this dataset, freely available within the nsROC package by the name interleukin6, can be found in Martínez-Camblor (2017). Particularly it includes true-positive (TP), false-positive (FP), true-negative (TN) and false-negative (FN) sizes for all cut-off points reported in each paper, resulting in 19 entries.

Figure 6 shows the summary ROC curve estimate from the 9 papers included, considering either a fixed-effects or a random-effects meta-analysis model (up and down, respectively). The optimal point of the curve in the Youden index sense is displayed, as well as the area under the curve. In this case, the curve does not vary much when the variability between studies is taken into account, reporting similar AUC ( 0.772 and 0.788 , respectively) as a consequence. In addition, both estimates seem to be below most of the interpolated curves they come from; that is because the weights for the 
ROC curve at time 4000 (Cox method)

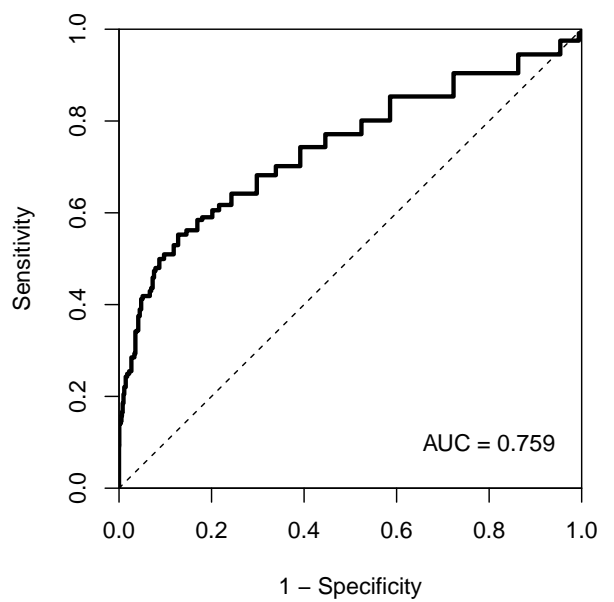

ROC curve at time 4000 (Weighted KM method with normal kernel)

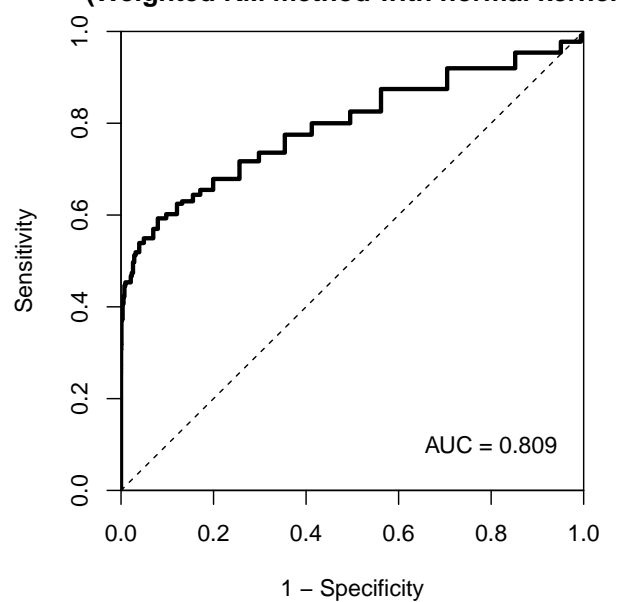

ROC curve at time 4000 (KM method)

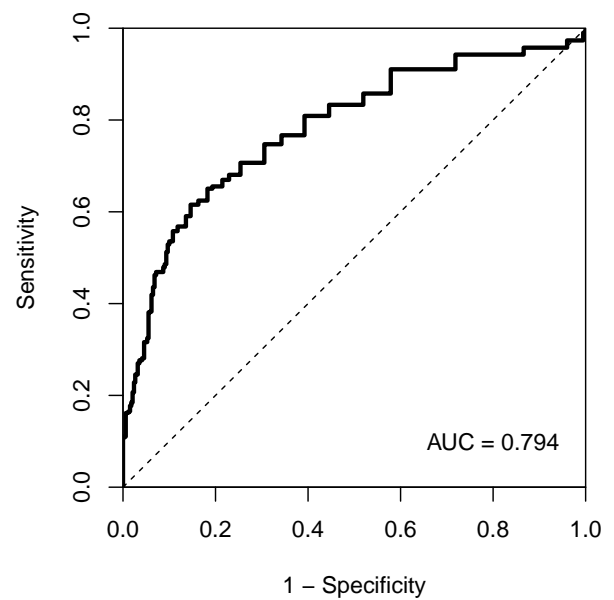

ROC curve at time 4000 (Weighted KM method with uniform kernel)

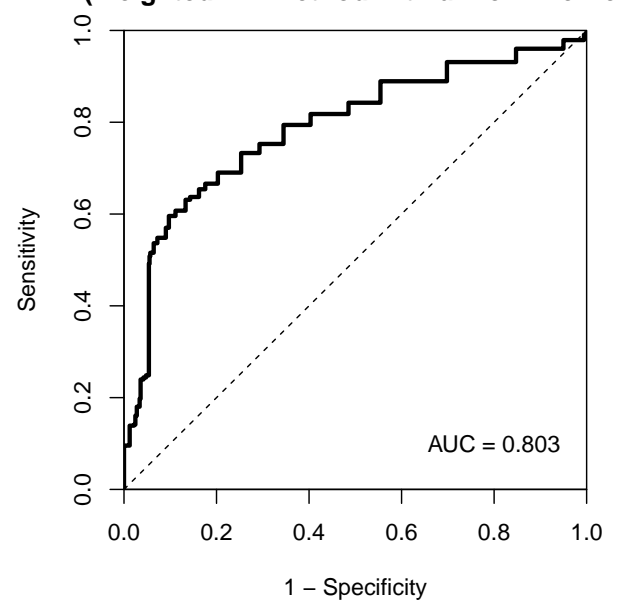

Figure 5: Time-dependent ROC curve estimate using "Cox", "KM" (top) and "wKM" method with normal kernel and bandwidth $h=1$ and with uniform kernel and $h=0.5$ (bottom), respectively.

study number 9 (with an interpolate ROC curve close to diagonal) are the largest ones in the interval $(0,0.5)$. As it can be seen in the bottom-right plot of Figure 6, the FPR interval with higher inter-study variability is $(0,0.2)$.

The code computed, using the metaRoc function, is listed below:

data(interleukin6)

output1 <- metaROC(interleukin6, plot.Author = TRUE)

\#> Number of papers included in meta-analysis: 9

\#> Model considered: fixed-effects

\#> The area under the summary ROC curve (AUC) is 0.772 .

\# The optimal specificity and sensitivity (in the Youden index sense) for summary

\#> ROC curve are 0.7 and 0.76 , respectively.

points(1-output1\$youden.index[1], output1\$youden.index[2], pch = 16, col = 'blue')

output $2<-$ metaROC(interleukin6, model = "random-effects", plot. Author = TRUE, plot. inter. var $=$ TRUE)

\#> Number of papers included in meta-analysis: 9

\#> Model considered: random-effects

\#> The area under the summary ROC curve (AUC) is 0.788 .

\#> The optimal specificity and sensitivity (in the Youden index sense) for summary

\#> ROC curve are 0.701 and 0.763 , respectively.

points (1-output2\$youden.index[1], output2\$youden.index[2], pch $=16$, col $={ }^{\prime} b l u e^{\prime}$ ) 
ROC curve (fixed-effects model)

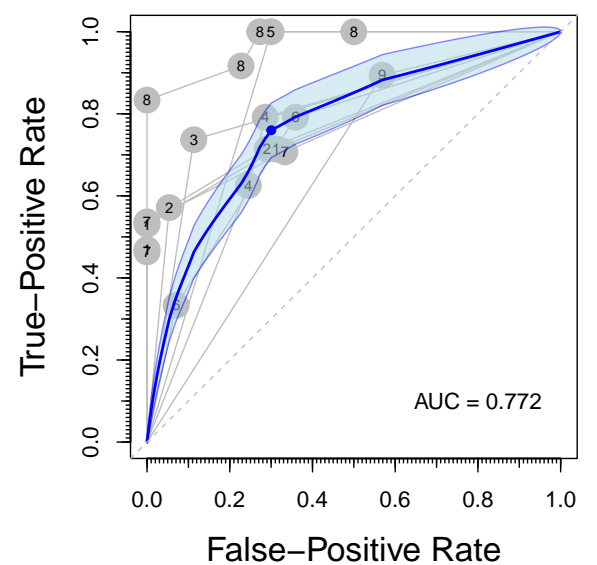

ROC curve (random-effects model)

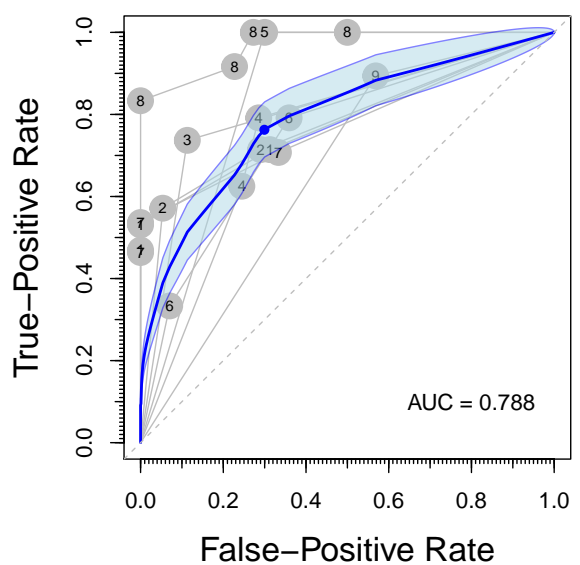

Inter-study variability

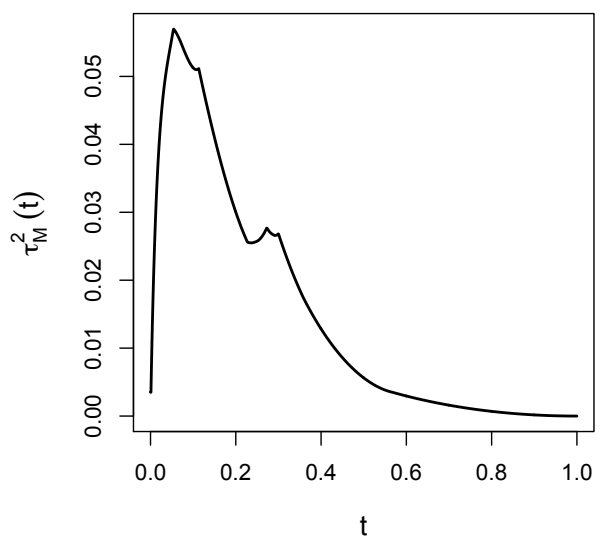

Figure 6: Summary ROC curve estimate considering a fixed-effects (top) and a random-effects metaanalysis model (bottom), respectively. Bottom-right, inter-study variability estimate of summary ROC curve reported by a random-effects model.

\section{Summary}

This article introduces the usage of the R package nsROC for analyzing ROC curves. In particular, the package contains the following new techniques:

- point ROC curve non-standard estimation implementing the generalization proposed by Martínez-Camblor et al. (2017) [gROC function];

- confidence bands construction by three different methods: two of them are non-parametric (Jensen et al. (2000) and Martínez-Camblor et al. (2018)) and the other one is based on the binormal model (Demidenko (2012)) [RoCbands function];

- time-dependent ROC curve estimation, dealing with the presence of censored data respect to the time-dependent response variable following Martínez-Camblor et al. (2016) procedure [cdROC function];

- meta-analysis, implementing the methods proposed by Martínez-Camblor (2017), covering both fixed and random-effects model considering all the points of the curve reported in each study [metaROC function];

- comparison of several ROC curves using different procedures, among which the ones based on usual tests to compare distribution functions proposed by Martínez-Camblor et al. (2011) and Martínez-Camblor et al. (2013) stand out. Not only the usual tests can be performed, but the user can define any other by the input parameters in the compareROCdep and compareROCindep functions. 
In spite of the popularity of R packages about ROC curves dealing with some of the most important analyses related to this tool, nsROC includes some algorithms which had not been computed to date in order to address some of those standard analyses (such as time-dependent ROC curve estimation and comparison between curves) and others totally new such as the generalized ROC curve estimation and non-parametric procedure for meta-analysis. Any of these particular techniques had been addressed earlier, excluding the usual estimation of the curve, the weighted Kaplan-Meier method to deal with the presence of censored data in time-dependent ROC curves estimation, and the Venkatraman and DeLong approaches to compare diagnostic accuracies of two tests.

The following table indicates which functions in the package can be used for different options of side of the ROC curve. In addition to this, it should be mentioned that cdROC function estimates a time-dependent ROC based on cumulative sensitivity and dynamic specificity definitions, which are ultimately related to right-sided ROC curve. On the other hand, metaROC function includes directly the TP, FP, TN and FN as input parameters, and those may have been generated by any ROC curve approach, but it should be the same for all studies considered.

\begin{tabular}{l|l|l}
\hline \multicolumn{1}{c|}{ Right } & \multicolumn{1}{c}{ Left } & \multicolumn{1}{c}{ Both } \\
\hline gROC & gROC & gROC \\
ROCbands & ROCbands(method=="PSN") \\
compareROCdep & $\begin{array}{l}\text { compareROCdep(method!="VK") } \\
\text { compareROCindep }\end{array}$ & \\
\hline
\end{tabular}

Table 7: Options of side of the ROC curve for different functions of the nsROC package

\section{Acknowledgements}

The authors acknowledge support by the Grants MTM2015-63971-P and MTM2014-55966-P from the Spanish Ministerio of Economía y Competitividad and by FC-15-GRUPIN14-101 from the Principado de Asturias.

\section{Bibliography}

K. P. Bennett and O. L. Mangasarian. Robust linear programming discrimination of two linearly inseparable sets. Optimization Methods and Software, 1(1):23-34, 1992. URL https://doi .org/10. 1080/10556789208805504. [p63]

P. Blanche. timeROC: Time-Dependent ROC Curve and AUC for Censored Survival Data, 2015. URL https://CRAN.R-project.org/package=timeROC. R package version 0.3. [p56]

P. Blanche, J.-F. Dartigues, and H. Jacqmin-Gadda. Estimating and comparing time-dependent areas under receiver operating characteristic curves for censored event times with competing risks. Statistics in Medicine, 32(30):5381-5397, 2013. URL https://doi . org/10.1002/sim. 5958. [p59]

T. Cai. Semi-parametric ROC regression analysis with placement values. Biostatistics, 5(1):45-60, 2004. URL https://doi.org/10.1093/biostatistics/5.1.45. [p56]

E. R. DeLong, D. M. DeLong, and D. L. Clarke-Pearson. Comparing the Areas under Two or More Correlated Receiver Operating Characteristic Curves: A Nonparametric Approach. Biometrics, 44(3): 837-845, 1988. URL https://doi.org/10.2307/2531595. [p61,62]

E. Demidenko. Confidence intervals and bands for the binormal ROC curve revisited. Journal of Applied Statistics, 39(1):67-79, 2012. URL https: //doi .org/10. 1080/02664763.2011.578616. [p55, $58,59,64,70]$

R. DerSimonian and N. Laird. Meta-analysis in clinical trials. Controlled Clinical Trials, 7(3):177-188, 1986. URL https://doi.org/10.1016/0197-2456(86)90046-2. [p60]

R. Fluss, D. Faraggi, and B. Reiser. Estimation of the Youden Index and its Associated Cutoff Point. Biometrical Journal, 47(4):458-472, 2005. URL https://doi .org/10. 1002/bimj . 200410135. [p55]

L. Gonçalvez, A. Subtil, M. R. Oliveira, and P. de Zea Bermudez. ROC curve estimation: An overview. REVSTAT Statistical Journal, 12(1):1-20, 2014. [p55] 
T. H. Hamza, J. B. Reitsma, and T. Stijnen. Meta-Analysis of Diagnostic Studies: A Comparison of Random Intercept, Normal-Normal, and Binomial-Normal Bivariate Summary ROC Approaches. Medical Decision Making, 28(5):639-649, 2008. URL https://doi . org/10.1177/0272989X08323917. [p60]

P. Heagerty and Y. Zheng. Survival model predictive accuracy and ROC curves. Biometrics, 61(1): 92-105, 2005. URL https://doi.org/10.1111/j.0006-341X.2005.030814.x. [p55,59]

P. Heagerty, T. Lumley, and M. S. Pepe. Time-dependent ROC curves for censored survival data and a diagnostic marker. Biometrics, 56(2):337-344, 2000. URL https: //doi .org/10.1111/j.0006$341 \times .2000 .00337 . x .[p 55,59]$

P. J. Heagerty and packaging by Paramita Saha-Chaudhuri. survivalROC: Time-dependent ROC curve estimation from censored survival data, 2013. URL https: //CRAN. R-project. org/package=survivalROC. $\mathrm{R}$ package version 1.0.3. [p56]

L. Horváth, Z. Horváth, and W. Zhou. Confidence bands for ROC curves. Journal of Statistical Planning and Inference, 138(6):1894-1904, 2008. URL https://doi .org/10.1016/j.jspi.2007.07.009. [p55]

A. Hoyer and O. Kuss. Meta-analysis for the comparison of two diagnostic tests to a common gold standard: A generalized linear mixed model approach. Statistical Methods in Medical Research, 27(5): 1410-1421, 2018. URL https://doi .org/10.1177/0962280216661587. [p60]

S. M. Iacus. sde: Simulation and Inference for Stochastic Differential Equations, 2016. URL https://CRAN. Rproject. org/package=sde. R package version 2.0.15. [p59]

K. Jensen, H.-H. Müller, and H. Schäfer. Regional confidence bands for ROC curves. Statistics in Medicine, 19(4):493-509, 2000. URL https: //doi .org/10.1002/(SICI) 1097-0258(20000229)19: 4<493: : AID-SIM352>3.0.C0;2-W. [p55, 58, 59, 64, 70]

L. Li, C. W. Department of Biostatistics, and The University of Texas MD Anderson Cancer Center. tdROC: Nonparametric Estimation of Time-Dependent ROC Curve from Right Censored Survival Data, 2016. URL https://CRAN.R-project.org/package=tdROC. R package version 1.0. [p59]

L. Li, T. Greene, and B. Hu. A simple method to estimate the time-dependent receiver operating characteristic curve and the area under the curve with right censored data. Statistical Methods in Medical Research, 27(8):2264-2278, 2018. URL https://doi .org/10.1177/0962280216680239. [p59, 60]

M. Lopez-Raton and M. X. Rodriguez-Alvarez. OptimalCutpoints: Computing optimal cutpoints in diagnostic tests, 2014. URL https: //CRAN. R-project. org/package=0ptimalCutpoints. R package version 1.1-3. [p56]

S. Macskassy, F. Provost, and S. Rosset. ROC Confidence Bands: An Empirical Evaluation. Proceedings of the 22nd international conference on machine learning, pages 537-544, 2005. URL https: //doi .org/ 10.1145/1102351.1102419. [p58]

P. Martínez-Camblor. Fully non-parametric receiver operating characteristic curve estimation for random-effects meta-analysis. Statistical Methods in Medical Research, 26(1):5-20, 2017. URL https: //doi.org/10.1177/0962280214537047. [p56, 60, 68, 70]

P. Martínez-Camblor and N. Corral. A general bootstrap algorithm for hypothesis testing. Journal of Statistical Planning and Inference, 142(2):589-600, 2012. URL https://doi.org/10.1016/j.jspi. 2011.09.003. [p61]

P. Martínez-Camblor and J. C. Pardo-Fernández. Parametric estimates for the receiver operating characteristic curve generalization for non-monotone relationships. Statistical Methods in Medical Research, (Published online), 2017. URL https://doi .org/10.1177/0962280217747009. [p57]

P. Martínez-Camblor, C. Carleos, and N. Corral. Powerful nonparametric statistics to compare k independent ROC curves. Journal of Applied Statistics, 38(7):1317-1332, 2011. URL https://doi . org/10.1080/02664763.2010.498504. [p56, 61, 62,70]

P. Martínez-Camblor, C. Carleos, and N. Corral. General nonparametric ROC curve comparison. Journal of the Korean Statistical Society, 42(1):71-81, 2013. URL https://doi .org/10.1016/j. jkss. 2012.05.002. [p56, 61,70]

P. Martínez-Camblor, G. F-Bayón, and S. Pérez-Fernández. Cumulative/dynamic ROC curve estimation. Journal of Statistical Computation and Simulation, 86(17):3582-3594, 2016. URL https: //doi.org/10.1080/00949655.2016.1175442. [p59,70] 
P. Martínez-Camblor, N. Corral, C. Rey, J. Pascual, and E. Cernuda-Morollón. Receiver operating characteristic curve generalization for non-monotone relationships. Statistical Methods in Medical Research, 26(1):113-123, 2017. URL https://doi .org/10.1177/0962280214541095. [p56, 57,70]

P. Martínez-Camblor, S. Pérez-Fernández, and N. Corral. Efficient nonparametric confidence bands for receiver operating-characteristic curves. Statistical Methods in Medical Research, 27(6):1892-1908, 2018. URL https://doi .org/10.1177/0962280216672490. [p55, 58, 59, 64, 70]

L. E. Moses, D. Shapiro, and B. Littenberg. Combining independent studies of a diagnostic test into a summary ROC curve: Data-analytic approaches and some additional considerations. Statistics in Medicine, 12(14):1293-1316, 1993. URL https://doi.org/10.1002/sim.4780121403. [p60]

D. Mossman. Three-way ROCs. Medical Decision Making, 19(1):78-89, 1999. URL https://doi .org/10. 1177/0272989X9901900110. [p55]

M. S. Pepe. The Statistical Evaluation of Medical Tests for Classification and Prediction. Oxford: Oxford University Press, 2003. ISBN 9780198509844. [p55]

S. Pérez-Fernández. nsROC: Non-Standard ROC Curve Analysis, 2018. URL https: //CRAN. R-project. org/package=nsRoc. R package version 1.1. [p56]

E. Peter. fbroc: Fast Algorithms to Bootstrap Receiver Operating Characteristics Curves, 2016. URL https: //CRAN.R-project. org/package=fbroc. R package version 0.4.0. [p56]

R. D. Riley, P. C. Lambert, and G. Abo-Zhaid. Meta-analysis of individual participant data: rationale, conduct, and reporting. British Medical Journal, 340, 2010. URL https://doi .org/10.1136/bmj .c221. [p60]

X. Robin, N. Turck, A. Hainard, N. Tiberti, F. Lisacek, J.-C. Sanchez, M. Müller, and S. Siegert. pROC: Display and Analyze ROC Curves, 2018. URL https: //CRAN. R-project. org/package=pROC. R package version 1.13.0. [p56]

M. X. Rodríguez-Álvarez, P. G. Tahoces, C. Cadarso-Suárez, and M. J. Lado. Comparative study of ROC regression techniques - Applications for the computer-aided diagnostic system in breast cancer detection. Computational Statistics and Data Analysis, 55(1):888-902, 2011. URL https: //doi.org/10.1016/j.csda.2010.07.018. [p56]

C. M. Rutter and C. A. Gatsonis. A hierarchical regression approach to meta-analysis of diagnostic test accuracy evaluations. Statistics in Medicine, 20(19):2865-2884, 2001. URL https://doi . org/10. 1002/sim. 942. [p60]

M. C. Sachs. plotROC: Generate Useful ROC Curve Charts for Print and Interactive Use, 2018. URL https://CRAN.R-project.org/package=plotRoc. R package version 2.2.1. [p56]

I. Schiller and N. Dendukuri. HSROC: Meta-Analysis of Diagnostic Test Accuracy when Reference Test is Imperfect, 2015. URL https://CRAN. R-project. org/package=HSROC. R package version 2.1.8. [p56]

T. Sing, O. Sander, N. Beerenwinkel, and T. Lengauer. ROCR: Visualizing the Performance of Scoring Classifiers, 2015. URL https://CRAN. R-project. org/package=ROCR. R package version 1.0-7. [p56]

S. Steinhauser, M. Schumacher, and G. Rücker. Modelling multiple thresholds in meta-analysis of diagnostic test accuracy studies. BMC Medical Research Methodology, 16(97):1-15, 2016. URL https://doi.org/10.1186/s12874-016-0196-1. [p60]

T. M. Therneau. survival: Survival Analysis, 2018. URL https://CRAN.R-project.org/package= survival. R package version 2.43-3. [p60]

T. M. Therneau and P. M. Grambsch. Modeling Survival Data: Extending the Cox Model. Springer, New York, 2000. ISBN 9780387987842. [p68]

E. Venkatraman. A permutation test to compare receiver operating characteristic curves. Biometrics, 56 (4):1134-1138, 2000. URL https://doi.org/10.1111/j.0006-341X.2000.01134.x. [p56, 61, 62]

E. Venkatraman and C. B. Begg. A distribution-free procedure for comparing receiver operating characteristic curves from a paired experiment. Biometrika, 83(4):835-848, 1996. URL https://doi . org/10.1093/biomet/83.4.835. [p56,61]

X.-H. Zhou, N. A. Obuchowski, and D. K. McClish. Statistical Methods in Diagnostic Medicine. Wiley, 2002. ISBN 9780471347729. URL https://doi.org/10.1002/9780470317082. [p55] 
Sonia Pérez Fernández

Department of Statistics and Operational Research and Mathematics Didactics University of Oviedo

Spain

perezsonia@uniovi.es

Pablo Martínez Camblor

The Dartmouth Institute for Health Policy and Clinical Practice

Geisel School of Medicine at Dartmouth

Hanover, NH, USA

Pablo. Martinez. Camblor@Dartmouth. edu

Peter Filzmoser

Institute of Statistics and Mathematical Methods in Economics

Vienna University of Technology

Austria

P.Filzmoser@tuwien.ac.at

Norberto Corral

Department of Statistics and Operational Research and Mathematics Didactics University of Oviedo

Spain

norbert@uniovi.es 


\title{
addhaz: Contribution of Chronic Diseases to the Disability Burden Using R
}

by Renata Tiene de Carvalho Yokota, Caspar WN Looman, Wilma Johanna Nusselder, Herman Van Oyen and Geert Molenberghs

\begin{abstract}
The increase in life expectancy followed by the burden of chronic diseases contributes to disability at older ages. The estimation of how much chronic conditions contribute to disability can be useful to develop public health strategies to reduce the burden. This paper introduces the R package addhaz, which is based on the attribution method (Nusselder and Looman, 2004) to partition disability into the additive contributions of diseases using cross-sectional data. The R package includes tools to fit the additive hazard model, the core of the attribution method, to binary and multinomial outcomes. The models are fitted by maximizing the binomial and multinomial log-likelihood functions using constrained optimization. Wald and bootstrap confidence intervals can be obtained for the parameter estimates. Also, the contribution of diseases to the disability prevalence and their bootstrap confidence intervals can be estimated. An additional feature is the possibility to use parallel computing to obtain the bootstrap confidence intervals. In this manuscript, we illustrate the use of addhaz with several examples for the binomial and multinomial models, using the data from the Brazilian National Health Survey, 2013.
\end{abstract}

\section{Introduction}

The increase in longevity observed worldwide is usually followed by the burden of chronic diseases, which are among the leading causes of disability late in life (Beard et al., 2016). Disability has become a public health priority due to its adverse effects on health outcomes and quality of life, resulting in increased costs of health care (Yang et al., 2014). Therefore, the identification of which chronic diseases are the main contributors to the disability burden plays an important role in the formulation of public health response to population aging (Klijs et al., 2011).

Although prospective studies are better suited to establish the causal relationship between chronic diseases and disability, they are costly and usually with limited sample size. Alternatively, crosssectional data has been widely used to investigate the association of chronic diseases and disability. Among the methods based on cross-sectional data, the attribution method proposed by Nusselder and Looman (2004) has the advantage of partitioning the disability prevalence into the additive contributions of chronic diseases, taking into account multimorbidity and that disability can be present even in the absence of chronic diseases. The method was originally proposed for binary outcomes, but it was recently extended to multicategory response variables (Yokota et al., 2017) and it is based on the binomial and multinomial additive hazard models, respectively. The use of non-canonical link functions in the models imposes a constraint on the linear predictor, which limits the use of standard statistical software to fit the models, such as glm in R or proc GLM in SAS (SAS Institute Inc., 2008). Despite this practical difficulty, the attribution method for binary outcomes has been widely used previously with data from the Netherlands (Nusselder and Looman, 2004; Klijs et al., 2011), Belgium (Nusselder et al., 2005; Yokota et al., 2015b), Germany (Strobl et al., 2013), China (Chen et al., 2013), and Brazil (Yokota et al., 2016), owing to the development of the software in R to fit the binomial model and to estimate the contribution of diseases to the disability prevalence by Nusselder and Looman (2010) for non-R users, which is available upon request to the authors (w.nusselder@erasmusmc.nl).

In this manuscript we present the $R$ package addhaz, which is an extension of the $R$ software developed by Nusselder and Looman (2010), offering an open-source implementation of the binomial and multinomial additive hazard models. The R functions can also be used to calculate the contribution of chronic diseases to the disability burden for both models.

This paper is structured as follows. In Section 2, a brief description of the attribution method is presented, followed by the definition of the binomial and multinomial additive hazard models. Section 3 introduces some features and options of addhaz. The existing alternative software to fit the binomial and multinomial models is discussed in Section 4 . Examples of how to use the $\mathrm{R}$ functions to fit the models and to estimate contributions are shown in Section 5, using the data of the 2013 Brazilian National Health Survey (BNHS). The main advantages and disadvantages of the attribution method and addhaz are discussed in Section 6. Finally, conclusions and recommendations for future research are outlined in Section 7. 


\section{Attribution method}

Analogous to the mortality analysis, in which a single disease is assigned as underlying cause of death in the death certificate, the attribution method aims to assess the probability that a single reported disease was the cause of disability in a survey, taking into account that individuals can report more than one disease (multimorbidity) and that disability can be present without any reported diseases (Nusselder and Looman, 2004, 2010).

In the attribution method, the disability that is not associated with any disease included in the analysis is attributed to "background". The background can represent the effect of age-related losses in functioning; underreporting and underdiagnosed diseases; and other causes of disability that were not included in the survey or analysis. More details about the attribution method can be found elsewhere (Nusselder and Looman, 2004, 2010).

The following assumptions are required to fit the binomial and multinomial additive hazard models to cross-sectional data: (i) disability is caused by the diseases that are still present in the time of the survey and the background; (ii) the estimated cross-sectional cumulative rates reflect the transition rates that would have been estimated with longitudinal data (stationarity assumption); (iii) the recovery rate is zero; (iv) the ratio of the cause-specific cumulative rates to the overall cumulative rate is constant over time (proportionality assumption); (v) the start of the time (age) at risk to become disabled is the same for all diseases; (vi) individuals from the same age group are exposed to the same cumulative rate of disability for background; (vii) diseases and background act as independent competing causes of disability (Nusselder and Looman, 2004, 2010).

\section{Binomial additive hazard model}

For binary outcomes, the binomial additive hazard model is defined as:

$$
\begin{aligned}
& y_{i} \sim \operatorname{Bernoulli}\left(\pi_{i}\right) \\
& \pi_{i}=1-\exp \left(-\eta_{i}\right) \\
& \eta_{i}=\alpha_{a_{i}}+\sum_{d=1}^{m} \beta_{d} X_{d i}
\end{aligned}
$$

where $y_{i}$ is the binary disability outcome; $\pi_{i}$ is the disability probability for individual $i ; \eta_{i}$ is the linear predictor representing the overall cumulative rate (or cumulative hazard) of disability for individual $i$; $a_{i}$ denotes the age group of individual $i$ (with $f$ age groups, $a_{i}$ can only get values between $1, \ldots, f$ ); $\alpha_{a}$ is the cumulative rate of disability for background by age group $a(a=1, \ldots, f) ; \beta_{d}$ is the cumulative rate of disability (or disabling impact) for disease $d(1, \ldots, m)$; and $X_{d i}$ is the indicator variable for disease $d$ and individual $i$.

A linear inequality constraint is applied to the linear predictor $\left(\eta_{i} \geq 0\right)$ to ensure that $\pi_{i}$ lies between $(0,1)$. The standard errors $(S E)$ for the regression coefficients are estimated based on the inverse of the observed information matrix. The 95\% Wald confidence intervals (Wald CI) can be obtained using the standard errors described above (Wald CI) as showed in 2 or via bootstrapping (Efron and Tibshirani, 1994).

$$
\begin{aligned}
& 95 \% \text { Wald CI }=\widehat{\alpha}_{a} \pm 1.96(\widehat{S E}) \\
& 95 \% \text { Wald CI }=\widehat{\beta}_{d} \pm 1.96(\widehat{S E})
\end{aligned}
$$

\section{Multinomial additive hazard model}

The multinomial additive hazard model is an extension of the binomial model:

$$
\begin{aligned}
y_{i j} & \sim \operatorname{Multinomial}\left(1, \Pi_{i}\right) \\
\pi_{i j} & =\left[1-\exp \left(-\sum_{q=1}^{c} \eta_{i q}\right)\right]\left(\frac{\eta_{i j}}{\sum_{q=1}^{c} \eta_{i q}}\right) \\
\eta_{i j} & =\alpha_{a_{i} j}+\sum_{d=1}^{m} \beta_{d j} X_{d i}
\end{aligned}
$$

where $y_{i j}$ is the multinomial response variable (disability) with one independent observation and vector of disability probabilities $\Pi_{i}=\left(\pi_{i 0}, \ldots, \pi_{i j}, \ldots, \pi_{i c}\right)$ per individual $i ; \pi_{i j}$ is the probability of disability category $j$ for individual $i ; \eta_{i j}$ is the linear predictor (overall cumulative rate) for disability category $j$ and individual $i$; $a_{i}$ denotes the age group of individual $i$ (with $f$ age groups, $a_{i}$ can only 
get values between $1, \ldots, f) ; \alpha_{a j}$ is the cumulative rate of disability category $j$ for background by age group $a(a=1, \ldots, f) ; \beta_{d j}$ is the cumulative rate of disability category $j$ or disabling impact for disease $d(1, \ldots, m)$; and $X_{d i}$ is the indicator variable for disease $d$ and individual $i$.

Besides the inequality constraint in the linear predictor $\eta_{i j} \geq 0$, an additional constraint is required: $\sum_{j=1}^{c} \pi_{i j}<1$, to ensure that all $\pi_{i j}>0$. Similar to the binomial case, the standard errors are estimated by the inverse of the observed information matrix and the $95 \%$ Wald CI and bootstrap percentile confidence intervals (bootstrap CI) can be obtained using addhaz.

\section{Contribution of chronic diseases and background to the disability prevalence}

The attribution of disability to chronic diseases depends on the disease prevalence $\left(X_{d}\right)$ and the disabling impacts of the diseases $\left(\beta_{d}\right.$ and $\beta_{d j}$ ) (Nusselder and Looman, 2004, 2010). The contribution of chronic diseases and background to the disability prevalence can be calculated in five steps for both binary and multicategory response variables.

\section{Binary case}

For the binary case, the cause-specific disability probabilities for individual $i$ and each chronic condition $\left(\widehat{D}_{d i}\right)$ and the background $\left(\widehat{B}_{i}\right)$ are estimated based on the proportionality assumption, analogous to the competing risks setting in mortality analysis (Manton and Stallard, 1984; Chiang, 1991):

$$
\begin{aligned}
& \widehat{D}_{d i}=\widehat{\pi}_{i}\left(\frac{\widehat{\beta}_{d} X_{d i}}{\widehat{\eta}_{i}}\right) \\
& \widehat{B}_{i}=\widehat{\pi}_{i}\left(\frac{\widehat{\alpha}_{a i}}{\widehat{\eta}_{i}}\right)
\end{aligned}
$$

Next, the number of disabled individuals by each disease $\left(\widehat{N}_{d}\right)$ and background $\left(\widehat{N}_{b}\right)$ are estimated as:

$$
\begin{aligned}
& \widehat{N}_{d}=\sum_{i=1}^{n} \widehat{D}_{d i} \\
& \widehat{N}_{b}=\sum_{i=1}^{n} \widehat{B}_{i}
\end{aligned}
$$

The absolute contribution of each disease $\left(\widehat{A C}_{d}\right)$ and background $\left(\widehat{A C}_{b}\right)$ to the total disability prevalence is obtained by:

$$
\begin{aligned}
& \widehat{A C}_{d}=\frac{\widehat{N}_{d}}{n} \\
& \widehat{A C}_{b}=\frac{\widehat{N}_{b}}{n}
\end{aligned}
$$

The absolute contribution of background and diseases defined above sum to the disability prevalence $(\widehat{P})$ :

$$
\widehat{P}=\widehat{A C}_{b}+\sum_{d=1}^{m} \widehat{A C}_{d}
$$

Finally, the relative contribution of diseases $\left(\widehat{R C}_{d}\right)$ and background $\left(\widehat{R C}_{b}\right)$ to the disability prevalence is estimated by:

$$
\begin{aligned}
& \widehat{R C}_{d}=\frac{\widehat{A C}_{d}}{\widehat{P}} \\
& \widehat{R C}_{b}=\frac{\widehat{A C}_{b}}{\widehat{P}}
\end{aligned}
$$

The relative contributions $\left(\widehat{R C}_{d}\right.$ and $\left.\widehat{R C}_{b}\right)$ sum to 1 .

\section{Multinomial case}

Analogous to the binomial case, the contribution of chronic diseases to the disability prevalence for multinomial outcomes can be obtained in five steps for each category $j$ of the outcome: 